\title{
Euclidean mirrors: enhanced vacuum decay from reflected instantons
}

\author{
Ibrahim Akal, ${ }^{1}$ Gudrid Moortgat-Pick, ${ }^{2}$ \\ ${ }^{1}$ Theory Group, Deutsches Elektronen-Synchrotron DESY, Hamburg, Germany \\ ${ }^{2}$ University Hamburg, II. Institute for Theoretical Physics, Hamburg, Germany \\ E-mail: ibrahim.akal@desy.de, gudrid.moortgat-pick@desy.de
}

\begin{abstract}
We study the tunneling of virtual matter-antimatter pairs from the quantum vacuum in the presence of a spatially uniform temporal electric background composed of of a strong slow field superimposed with a weak rapid field. After analytic continuation to Euclidean spacetime we obtain from the instanton equations two critical points. While one of them is the closing point of the instanton path, the other serves as an Euclidean mirror which reflects and squeezes the instanton. It is this reflection and shrinking which is responsible for an enormous enhancement of the vacuum pair production rate. We discuss how important features of this mechanism can be analysed and understood via such a rotation in the complex plane. Consistent with previous studies, we consider certain examples where we apply weak fields with a distinct pole structure in order to show that the reflection takes place exactly at the poles. We also discuss the effect of possible sub-cycle structures. We extend this reflection picture to fields which have no poles present and illustrate the effective reflections with explicit examples. An additional field strength dependence for the rate occurs in such cases. We analytically compute the characteristic threshold for this mechanism given by the critical combined Keldysh parameter. We discuss significant differences between these two types of fields. For various backgrounds, we present the contributing instantons and perform analytical computations for the corresponding rates treating both fields nonperturbatively. The validity of the results is confirmed by numerical computations. Considering different profiles for the strong field, we also discuss its impact on the critical combined Keldysh parameter.
\end{abstract}




\section{Contents}

1 Introduction $\quad 1$

2 Tunneling instantons 3

3 Effect of inhomogeneities 5

4 Superimposed electric field $\quad 8$

$\begin{array}{llr}5 & \text { Reflected instantons } & 9\end{array}$

$\begin{array}{llr}6 & \text { Fields with poles } & 12\end{array}$

$\begin{array}{lll}7 & \text { Fields without poles } & 19\end{array}$

8 Critical inhomogeneity 33

$\begin{array}{lll}9 & \text { Summary } & 37\end{array}$

$\begin{array}{ll}\text { A Improved mirrors: } \delta \text { correction } & 39\end{array}$

B Critical Keldysh parameter: $\Delta$ correction $\quad 41$

$\begin{array}{ll}\text { C Non-static strong field } & 43\end{array}$

$\begin{array}{ll}\text { D Additional figures } & 46\end{array}$

\section{Introduction}

The decay of the quantum vacuum against the production of matter-antimatter pairs in the presence of a spatially uniform static electric background has been predicted long time ago by Schwinger [1]. The rate in the weak field and weak coupling limit for this so-called Schwinger effect is given by

$$
\mathcal{R} \simeq \frac{(e E)^{2}}{(2 \pi)^{3}} e^{-\mathcal{W}_{0}}
$$

with $e$ being the particle charge and $E$ the electric field strength, respectively. The prefactor counts for the quantum fluctuation and the characteristic exponential signals the quantum mechanical tunneling behaviour of this purely nonperturbative ${ }^{1}$ absorptive process. The

\footnotetext{
${ }^{1}$ The perturbative treatment in $E, e$ fails, see e.g. [2].
} 
damping factor has the following dependence on the field strength,

$$
\mathcal{W}_{0}=\pi \frac{E_{\mathrm{S}}}{E}
$$

where $E_{\mathrm{S}}:=m^{2} / e$, with $m$ being the particle mass, denotes the characteristic Schwinger limit beyond which the vacuum decay is expected to become significant. Despite its elegant and relatively simple derivation in Quantum Electrodynamics (QED), vacuum pair production still could not yet be realised in the laboratory, mainly due to $E_{\mathrm{S}}=1.3 \times 10^{18}$ $\mathrm{V} / \mathrm{m}$ being extremely large.

However, significant efforts, on experimental as well as theoretical side, have been made in recent years that may bring vacuum pair production within experimental reach. For recent reviews we refer to $[3,4]$ and references therein. Strong field facilities with field strengths approaching $E \sim 10^{-3} E_{\mathrm{S}}$ (ELI) [5], or even higher, will be available within the next couple of years. Besides, there have been predicted highly promising scenarios in order to enhance the decay rate with temporally inhomogeneous electric fields. One of the most prominent is the so-called dynamically assisted mechanism [2,6-11]. Overlapping multiple pulsed fields [12] and optimisation of the field profile [13-16] can drive the rate higher as well. Besides, there appear remarkable effects if magnetic field components interact [17-19]. There are also substantial effects if thermalisation is involved [20-25]. Meanwhile, investigations of analogue condensed matter systems like graphene layers [26$31]$, semiconductors [32, 33] and semimetals [34] have become an active area.

The Schwinger effect is not necessarily restricted to QED. More general, it applies to quantum field theories (QFTs) containing a $U(1)$ gauge field. There may be also generalisations to non-Abelian gauge fields as for instance in Quantum Chromodynamics (QCD) [35]. Furthermore, in recent years there has been interesting progress on investigations from a much more fundamental point of view within holographic models to study, for instance, the effect of catastrophic instability [36-40], effects in confining gauge theories [41-48], and making even the connection to the recently proposed $\mathrm{ER}=\mathrm{EPR}$ conjecture $[49,50]$.

In this paper we will focus on the enormous enhancement of the decay rate via the dynamically assisted mechanism. This mechanism basically applies in an electric background that is assumed to be the superposition of a slowly varying strong field and a much faster varying weak field. A fully analytical treatment with such a combined electric background is in general difficult to perform. We utilise the semiclassical instanton approach to employ the reflection picture ${ }^{2}[6]$. According to this description the enhancement can be simply understood as instanton reflections in Euclidean spacetime. We show that many properties and characteristic features of this mechanism can be explained by means of such reflections. Particularly, we focus on the role of the assisting weak rapid field that, depending on its profile, has remarkable affects on the vacuum decay rate $[7,11,51]$. Based on geometrical considerations, we explain the origin for such significant differences.

The remaining part of the paper is structured as follows. In Sec. 2 we give a brief discussion of vacuum pair production in the worldline formalism and introduce the sta-

\footnotetext{
${ }^{2}$ It is important to note that the effect of dynamical assistance requires the nonperturbative treatment of the weak rapid field, see e.g. [2].
} 
tionary instanton equations. In Sec. 3 we briefly discuss the effects of temporal field inhomogeneities. Afterwards, in Sec. 4, we set up the general form of combined electric backgrounds we will consider in this paper. In Sec. 5 we work out in detail the instanton reflections. Using this reflection picture, we first study in Sec. 6 the type of weak fields which possess a distinct pole structure. In Sec. 7 we extend our investigations to fields without poles. We analytically compute the effective reflection points and exact critical Keldysh parameters for several field profiles. Sec. 8 includes discussion of the critical Keldysh parameter dependence on the strong field profile. In Sec. 9 we summarise and conclude.

Apps. A and B deal with explicit computations of the effective reflection and critical points for fields without poles. Based on geometric considerations, we show how to solve analytically the in general complicated transcendental equations to relevant order. In App. C we demonstrate the characteristic critical threshold dependence on the strong field with an explicit example. In App. D we have attached some additional figures for the sake of completeness.

\section{Tunneling instantons}

Let us begin with a brief discussion of vacuum decay in the presence of a classical electromagnetic field within the string-inspired [52] worldline formalism in quantum field theory $[53,54]$. Although this technique is completely different from Schwinger's original work, it leads in an elegant way to the correct rate even beyond the weak coupling regime [55]. According to [1], the probability for vacuum decay against the production of matterantimatter pairs is given by

$$
\mathcal{P}=1-e^{-2 \mathcal{R}}
$$

The rate $\mathcal{R}$ is determined by the imaginary part of the one-loop Euler-Heisenberg (EH) effective action $[1,56]$. One can analytically continue to Euclidean spacetime and integrate out the high energy degrees of freedom. Such an effective description will then be restricted to the regime $\omega \ll m$ where $\omega$ denotes the classical field frequency. In addition, the former step will imply that the decay rate will be determined by the real part of the corresponding effective action. In other words, we would resign the (Lorentzian) time direction via a rotation in the complex plane to obtain a real valued action describing the physics of the quantum vacuum.

For the present discussion, we focus on the weak coupling regime and neglect the contribution coming from the dynamical part $A_{\mu}$ of the vector field that is split into $A_{\mu}+\mathcal{A}_{\mu}$. The latter stands for the classical electromagnetic vector potential whose general shape is discussed further below. In this case, introducing the proper time $s$ [57] and applying the quantum mechanical path integral representation $[58,59]$ for the trace using position eigenstates [54] (paths in spacetime, so-called worldlines), one arrives at the following worldline representation

$$
\mathcal{R} \simeq \int_{0}^{\infty} \frac{d s}{s} e^{-m^{2} s} \int \mathcal{D} x(\tau) e^{-\int_{0}^{s} d \tau\left(\frac{\dot{x}^{2}(\tau)}{4}+i \mathcal{A} \cdot \dot{x}(\tau)\right)}
$$


in scalar QED (sQED) where $\mathcal{D} x$ denotes the path integration measure over the worldlines. Here, we may consider for simplifying reasons SQED, since the fermion spin does not play a decisive role [60]. Note that $x_{\mu}(\tau)$ satisfies the boundary condition $x_{\mu}(0)=x_{\mu}(s)$. Rescaling the worldline under $\tau=u s$ and performing a saddle-point analysis in $s$, one obtains over all closed paths, now specified by

$$
\begin{gathered}
\mathfrak{p}=\left\{x_{\mu} \mid x_{\mu}(0)=x_{\mu}(1)\right\}, \\
\mathcal{R} \simeq \int_{\mathfrak{p}} \mathcal{D} x(u) \sqrt{\frac{2 \pi}{m^{2} s_{0}}} \exp (-\mathcal{W}) .
\end{gathered}
$$

The stationary point is

$$
s_{0}=\frac{1}{m} \sqrt{\int_{0}^{1} d u \dot{x}^{2}(u)} .
$$

This method is applicable to the fermionic case as well [61]. The latter result is valid in the so-called large mass approximation (LMA),

$$
m^{2} s_{0} \gg 1 .
$$

The worldline action $\mathcal{W}$ consists of a kinetic part $\mathcal{W}_{\text {kin }}$ and an external part $\mathcal{W}_{\text {ext }}$,

$$
\mathcal{W} \stackrel{\text { weak }}{\simeq} \mathcal{W}_{\text {kin }}+\mathcal{W}_{\text {ext }}=m^{2} s_{0}+i \int_{0}^{1} d u \mathcal{A} \cdot \dot{x}(u) .
$$

Note that performing the integrations in the opposite order, i.e. first the integral for $x_{\mu}(u)$ and then the $s$ integration, will lead to the EH effective action on which Schwinger's original derivation is based on.

The $x_{\mu}(u)$ integration can be done using the method of steepest descents [55]. Expanding the worldline action in the fluctuations over the (worldline) instanton, i.e. $x_{\mu}(u) \rightarrow$ $x_{\mu}(u)+\delta x_{\mu}(u)$, this leads to

$$
\mathcal{R}=\frac{1}{\sqrt{\operatorname{Det} M}} e^{-\mathcal{W}_{0}}
$$

where

$$
\mathcal{W}_{0} \equiv \mathcal{W}[\text { instanton }]
$$

Note that $M_{\mu \nu}$ is the second order variation operator [55]. It is the expression in front of the exponential in (2.8) that leads to the given quantum fluctuation prefactor in (1.1). The general form of the prefactor for temporally or spatially inhomogeneous electric fields as a function of one spacetime coordinate has been derived in [62], evaluating the different determinants from the corresponding integrations, three in total, separately. Using the Gutzwiller trace formula, it has been shown that the total prefactor is encoded in a single determinant that is determined by the monodromy matrix [63]. Recently, it has been shown 
in detailed numerical computations that important features in the dynamically assisted mechanism are mainly unaffected by the prefactor [64]. For the present discussions we will only focus on the exponential $e^{-\mathcal{W}_{0}}$. Detailed studies regarding the corresponding fluctuation prefactors will be presented elsewhere [65]. Using the anti-symmetry of the field tensor $\mathcal{F}_{\mu \nu}=\partial_{\mu} \mathcal{A}_{\nu}-\partial_{\nu} \mathcal{A}_{\mu}$ one can show that

$$
\dot{x}^{2}=\text { constant } \equiv a^{2} .
$$

Thus, the closed (worldline) instanton path is determined by the following set of equations $[55]$

$$
m \ddot{x}_{\mu}=i a e \mathcal{F}_{\mu \nu} \dot{x}_{\nu}
$$

The complete solution including the fluctuation prefactor is exact $[55,66,67]$ for a quadratic action in $x_{\mu}(u)$ and approximate for actions with higher order dependence.

In the following we consider time dependent fields oriented in $\hat{x}_{3}$ direction. The instanton equations (2.11) from above then read as

$$
\begin{aligned}
& \ddot{x}_{4}=+\frac{i e a}{m} \partial_{4} \mathcal{A}_{3}\left(x_{4}\right) \dot{x}_{3}, \\
& \ddot{x}_{3}=-\frac{i e a}{m} \partial_{4} \mathcal{A}_{3}\left(x_{4}\right) \dot{x}_{4} .
\end{aligned}
$$

Furthermore, as mentioned before, to allow only real instanton solutions, we will consider electric fields which will be described by analytic even functions in time $t$. The corresponding vector potentials for such fields can be written in the form

$$
\mathcal{A}_{3}\left(x_{4}\right)=-i E F\left(x_{4}\right)
$$

where $F$ is assumed to be an odd real function. The complex $i$ in front guarantees that the instanton equations (2.12) will have real closed solutions $x_{\mu}$.

\section{Effect of inhomogeneities}

The effect of temporal electric field inhomogeneities in vacuum pair production has been studied extensively in the literature, e.g. cf. [6, 61, 68-73]. It has been shown that those field profiles can enhance the production rate even with field strengths far below the critical Schwinger limit $E_{\mathrm{S}}$. The intuitive physical picture is that the vacuum energy gap, which has to be overcome by the virtual pair, can effectively be lowered by the additional inhomogeneity in spacetime. This is in close analogy to atomic ionisation processes, e.g. cf. [74].

In the worldline formalism these modifications can be very elegantly illustrated with the help of the stationary instanton solutions. It has been shown that inhomogeneities of latter type tend to shrink the instanton which then leads to a larger pair production rate [61]. The technical reason for such an enhancement can therefore be obtained directly from the instanton equations. 
However, one should note that for arbitrary inhomogeneous fields, particularly for spatiotemporal type, the situation can be very complicated due to the increasing nonlinear structure of the underlying equations. Hence, it can be quite difficult to get some approximate information directly from the instanton equations. Effects of spatiotemporal fields have been recently studied [75-80]. In the following we will focus on various types of temporally inhomogeneous fields of the form we have discussed in the previous section. To work out the differences, we will briefly start with the instanton equations in a static electric field and show that those solve the circle equation. This is the most simple case where we can find a closed instanton path.
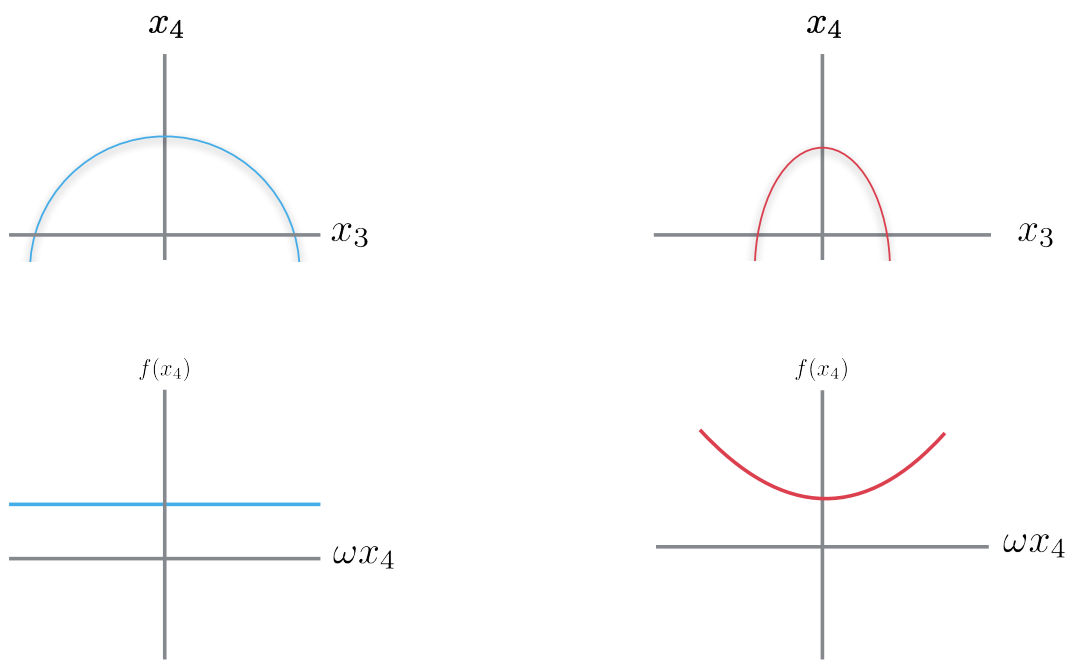

Figure 1. Effect of temporal field inhomogeneities: as an example, the comparison between a static and sinusoidal electric field is sketched. For the latter the bounded cosine function becomes after the rotation in the complex plane the (from above) unbounded hyperbolic cosine function (right). The static field remains static (left). Expected instanton paths around the closing point are depicted on top.

We begin with the following relations which we can directly read off from the following relevant equations,

$$
\dot{x}_{4}^{2}+\dot{x}_{3}^{2}=a^{2}, \quad \frac{\ddot{x}_{4}}{\dot{x}_{3}}=a / R, \quad \frac{\ddot{x}_{3}}{\dot{x}_{4}}=-a / R .
$$

$a$ is the constant kinematic invariant and $R=\frac{m}{e E}$. Since $\frac{a}{R}$ is constant, we can integrate the second and third equation in (3.1) to obtain the system

$$
\dot{x}_{4}=\frac{a}{R} x_{3}, \quad \dot{x}_{3}=-\frac{a}{R} x_{4} .
$$

Squaring the latter equations and applying the first relation in (3.1) afterwards, we obtain the circle equation

$$
x_{4}^{2}+x_{3}^{2}=R^{2} .
$$


Hence, the instanton for a static field must be a circle. This observation one may also guess just by looking on the equations in (3.1). In particular the right-hand side (RHS) of the last two equations is a fixed constant $\frac{a}{R}$. In other words, the ratio between the acceleration $\ddot{x}_{4}\left(\ddot{x}_{3}\right)$ in one direction and the velocity $\dot{x}_{3}\left(\dot{x}_{4}\right)$ in the remaining direction is constant. Kinematically, this situation is realised along a circle path. Hence, the electric field in Euclidean spacetime acts like a magnetic field leading to a circular instanton path.

If this static electric field is oriented in the $\hat{x}_{3}$ direction, i.e.

$$
\mathcal{A}_{3}\left(x_{4}\right)=-i E x_{4},
$$

the resulting circular path in the $\left(x_{3}, x_{4}\right)$ plane is described by

$$
x_{3}(u)=R \cos \left(\frac{e E}{m} a u\right), x_{4}(u)=R \sin \left(\frac{e E}{m} a u\right),
$$

with $a=2 \pi n R$ and radius $R=\frac{m}{e E}$ that follows due to the periodicity condition $\mathfrak{p}$ [55].

Note that the distance along the spatial $\hat{x}_{3}$ axis at time $x_{4}=0$ is

$$
x=2 R=\frac{2 m}{e E} .
$$

This result one can already obtain from simple energy conservation. Namely, the energy $x e E$ that is needed during delocalising the virtual pair to make it real along a distance $x$ has to be $2 \mathrm{~m}$. This is the relation from above. Note that if $x=2 \lambda_{c}$, where $\lambda_{c}$ denotes the usual Compton wavelength, this simple analysis brings us to the characteristic Schwinger limit $E_{\mathrm{S}}=\frac{m^{2}}{e}$, except the prefactor $\pi$, cf. (1.2). Therefore, the width of the effective energy gap between the excited particle states and the Dirac sea is naturally encoded in the spatial width ${ }^{3}$ of the instanton at zero (Euclidean) time.

Coming back to the circle solution, we find $n$ given in $a$ as the so-called winding number that counts for the number of times the Euclidean path is traversed. The higher order instanton contributions with $n>1$ correspond to the production of $n$ pairs [81, 82]. It is not clear whether this argument is justified for the case of strongly coupled nonAbelian gauge theories. For the circle instanton the LMA from (2.6) becomes immediately $E \ll E_{\mathrm{S}}$. For $n=1$ we find the previous evaluated action $\mathcal{W}_{0}$ from (1.2) (as the dominating contribution in the weak field limit). Taking in addition the fluctuation prefactor [55, 62] into account, the complete result for the vacuum decay probability reads

$$
\mathcal{R} \simeq \frac{(e E)^{2}}{(2 \pi)^{3}} \sum_{n=1}^{\infty} \frac{(-1)^{n+1}}{n^{2}} e^{-\pi n \frac{E_{\mathrm{S}}}{E}}
$$

which is precisely the original Schwinger formula. The first term is the rate from (1.1).

The situation for a non-static electric field is far more complicated. The system, one has to solve in this case, is given by

$$
\dot{x}_{4}^{2}+\dot{x}_{3}^{2}=a^{2}, \quad \frac{\ddot{x}_{4}}{\dot{x}_{3}}=f\left(x_{4}\right), \quad \frac{\ddot{x}_{3}}{\dot{x}_{4}}=-f\left(x_{4}\right) .
$$

\footnotetext{
${ }^{3}$ As we will discuss, fields with temporal inhomogeneities in spacetime lead in general to a substantial reduction of the tunneling barrier, i.e. $m^{*}<m$, which corresponds then to a smaller spatial width $x^{*}<x$ of the instanton trajectory at $x_{4}=0$.
} 
The constant RHS of the last two equations is now described by $\pm f\left(x_{4}\right)$. The function $f\left(x_{4}\right)$ is nothing but the analytic continuation (except the imaginary prefactor $-i$ ) of the physical electric field to Euclidean spacetime. Therefore, inhomogeneous electric fields with some oscillatory profile may become unbounded positive monotonic functions in the instanton equations, cf. lower right panel in Fig. 1. Let us make this a bit more concrete. For instance, the sinusoidal cosine becomes after the rotation in the complex plane the hyperbolic cosine function. This in addition brings the imaginary prefactor ${ }^{4}$ in front, which we have already discussed at the beginning. In other words, the complex exponential of cosine becomes the unbounded real exponential.

In this case one will find points where the acceleration in one direction may become much larger than the velocity in the other. The equations of such a system may have ellipselike solutions which can curve much stronger than the usual circle path. As a consequence, the size of the instanton can drastically be reduced for appropriate field parameters, e.g. sufficiently large temporal inhomogeneities [61]. From the previous discussion, this reduction would correspond to a smaller instanton extension $x^{*}$ at $x_{4}=0$ or smaller effective mass $m^{*}$, respectively, means that the tunneling barrier is reduced. Consequently, the rate for vacuum pair production in such background fields will be increased compared to the static field. Note that the latter remains static even after continuation to Euclidean spacetime, cf. lower left panel in Fig. 1.

However, the huge impact of temporal inhomogeneous electric fields on vacuum pair production is not only initiated by their unbounded shape in the instanton equations. Another property which arises, for instance, for bell-shaped inhomogeneous electric fields, as for instance for the single-pulse field, the so-called Sauter field, has major impact on the enhancement. Such field profiles show distinct pole structures which may be much more efficient on reshaping the instanton paths towards reduction. It is this reason why we think that, despite the differences regarding quantum interferences ${ }^{5}$, the temporal Sauter field leads to a stronger enhancement than the sinusoidal field where in contrast no poles are present, e.g. cf. [61]. The main purpose of this paper is to study the impact of such properties on the process of vacuum pair production, particularly, in the context of the dynamically assisted mechanism in spatially uniform but temporally inhomogenenous electric backgrounds.

\section{Superimposed electric field}

We consider a superimposed classical electric background which is described by

$$
\boldsymbol{E}(t)=(E f(t)+\tilde{E} g(t)) \hat{x}_{3} .
$$

\footnotetext{
${ }^{4}$ Note that it is this complex prefactor which makes the instanton solution real for the presently considered electric backgrounds.

${ }^{5}$ We do not consider interference effects in the present studies. Basically, those are encoded and manifest in the phase-space of the produced pairs, cf. e.g. [8, 51, 83-86]. The pole structure of the field is expected to be essential for their appearance, cf. [11]. For further sensitivity and optimisation studies those effects should be taken into account.
} 
The weak rapid field, $\propto \tilde{E}$ with frequency $\tilde{\omega}$, is described by an analytic function $g(t)$, whereas the the strong slow field, $\propto E$ with frequency $\omega$, is characterised by a function $f(t)$. The fields shall satisfy the hierarchies $E_{\mathrm{S}} \gg E \gg \tilde{E}$ and $m \gg \tilde{\omega} \gg \omega$, and both functions $f, g$ are assumed to be even in time $t$. As we have previously mentioned, the latter constraint we impose to obtain only real instanton solutions. Note that in general those can be complex as well, e.g. cf. [87]. The corresponding gauge potential after analytic continuation to Euclidean spacetime reads

$$
\mathcal{A}_{3}\left(x_{4}\right)=-i E F\left(x_{4}\right)-i \tilde{E} G\left(x_{4}\right) .
$$

Here $F\left(x_{4}\right)$ and $G\left(x_{4}\right)$ denote the corresponding odd functions that one obtains after the integration of the electric field functions $f(t)$ and $g(t)$, respectively. Inserting the temporal derivative of the gauge potential

$$
\partial_{4} \mathcal{A}_{3}\left(x_{4}\right)=-i\left(E F^{\prime}\left(x_{4}\right)+\tilde{E} G^{\prime}\left(x_{4}\right)\right)
$$

into the instanton equations, we find the following nonlinearly coupled system of differential equations

$$
\begin{aligned}
& \ddot{x}_{4}=+\frac{e a E}{m}\left(F^{\prime}\left(x_{4}\right)+\epsilon G^{\prime}\left(x_{4}\right)\right) \dot{x}_{3}, \\
& \ddot{x}_{3}=-\frac{e a E}{m}\left(F^{\prime}\left(x_{4}\right)+\epsilon G^{\prime}\left(x_{4}\right)\right) \dot{x}_{4},
\end{aligned}
$$

where $\epsilon:=\tilde{E} / E$ is introduced as a dimensionless parameter.

\section{$5 \quad$ Reflected instantons}

As already mentioned, in the following we will focus on the purely time-dependent enhancement in vacuum pair production, i.e. dynamically assisted (Schwinger) mechanism. It applies if $E_{\mathrm{S}} \gg E \gg \tilde{E}$ and $m \gg \tilde{\omega} \gg \omega$. The former condition corresponds to the limit $\epsilon \ll 1$. Introducing the so-called dimensionless Keldysh parameters,

$$
\gamma=\frac{m \omega}{e E}, \quad \tilde{\gamma}=\frac{m \tilde{\omega}}{e E},
$$

the latter frequency condition from above can be written as $\tilde{\gamma} / \gamma \gg 1$ where $\gamma, \tilde{\gamma} \ll E_{\mathrm{S}} / E$. The former strong field inhomogeneity parameter $\gamma$ interpolates between the adiabatic nonperturbative tunneling regime $(\gamma \ll 1)$ and the anti-adiabatic perturbative multi-photon regime $(\gamma \gg 1)[88]$. Note that $\tilde{\gamma}$ is the so-called combined Keldysh parameter that involves the strong field amplitude $E$ and the weak field frequency $\tilde{\omega}$. For $\tilde{\gamma} \ll 1$ the usual Schwinger mechanism is approached. One should note, that $\tilde{\gamma} \gg 1$ does not correspond to a pure perturbative multi-photon weak field. We will see, that in cases where the weak field $\propto \tilde{E}$ possesses a distinct pole structure, this parameter becomes the main quantity in the assisted mechanism ${ }^{6}$. Now, the second equation in (4.4) can be integrated as

$$
\dot{x}_{3}=-\frac{e a E}{m}\left(F\left(x_{4}\right)+\epsilon G\left(x_{4}\right)\right) .
$$

\footnotetext{
${ }^{6}$ We will discuss in detail that weak fields without a distinct pole structure lead in general to an additional $\epsilon$ dependence of the vacuum pair production rate, cf. Sec. 7 .
} 
Using the kinematic invariant $a=\sqrt{\dot{x}_{3}^{2}+\dot{x}_{4}^{2}}$, we may write the equation for $\dot{x}_{4}$. However, the integral is generally difficult to solve analytically because of its nonlinear dependence on the integration variable $x_{4}$. An effective reflection picture [6] will provide a simplified way to tackle this problem analytically, cf. e.g. [77].

Namely, since we are interested in the limit $\epsilon \ll 1$, we may omit the second term in (5.2). However, going back to the original equations (4.4), this is allowed as long the derivative $G^{\prime}\left(x_{4}\right)$ is sufficiently small. As soon as it becomes very large, which happens for sure at some pole, determined by

$$
1 / g\left(x_{4}^{\mathrm{p}}\right)=0
$$

one expects a substantial contribution from this term. For the moment, let us assume that the weak field has a distinct pole structure. Note that due to symmetry reasons, which apply for the specific field configurations considered in this paper, it is sufficient to do the present analysis with respect to the pole on the positive time axis, i.e. $+x_{4}^{\mathrm{p}}$. Thus, the weak field pole acts as an infinite wall where the instanton will be reflected with a nonvanishing velocity $\dot{x}_{4}$. Away from these Euclidean mirrors, as we call such reflection points, we can neglect the second terms in the brackets and integrate the instanton equations approximately as

$$
\begin{aligned}
& \dot{x}_{3} \approx-a \frac{\omega}{\gamma} F\left(x_{4}\right), \\
& \dot{x}_{4} \approx \pm a \sqrt{1-\left(\frac{\omega}{\gamma} F\left(x_{4}\right)\right)^{2}} .
\end{aligned}
$$

Note that the kinematic invariant $a$ provides the nontrivial coupling between $\dot{x}_{3}$ and $\dot{x}_{4}$. Since in the reflection points we will find $\dot{x}_{4} \neq 0$, the invariant $a$ is expected to be modified. This modification will be discussed soon. For $\epsilon \ll 1$ we then write the external part in the worldline action $\mathcal{W}$ as

$$
\mathcal{W}_{\mathrm{ext}}=i e \int_{0}^{1} d u \dot{x} \cdot \mathcal{A} \approx e E \int_{0}^{1} d u \dot{x}_{3}(u) F\left(x_{4}(u)\right) .
$$

Due to the instanton symmetry we can use the relation

$$
\int_{0}^{1} d u \dot{x}_{3}=4 \int_{0}^{x_{4}^{\mathrm{c}}} d x_{4} \frac{\dot{x}_{3}}{\dot{x}_{4}}
$$

where $x_{4}^{\mathrm{c}}$ is the closing point at the intersection between the first and second quarter (at $x_{3}=0$ ). This is allowed, since the derivatives $F^{\prime}, G^{\prime}$ are even functions (note that the electric background is assumed to be described by even functions). Therefore, the expected instanton path resulting from the stationary equations (5.4) will own the following symmetry

$$
\begin{aligned}
& x_{3} \rightarrow-x_{3}, \\
& x_{4} \rightarrow-x_{4} .
\end{aligned}
$$


Taking those aspects into account, we may proceed with the expression for the external part of the stationary worldline action,

$$
\mathcal{W}_{0, \text { ext }}=4 e E \int_{0}^{x_{4}^{\mathrm{c}}} d x_{4} \frac{-\frac{\omega}{\gamma} F\left(x_{4}\right) F\left(x_{4}\right)}{\sqrt{1-\left(\frac{\omega}{\gamma} F\left(x_{4}\right)\right)^{2}}},
$$

where $x_{4}^{\mathrm{c}}$ is the closing point in which the instanton has to be closed on the $\hat{x}_{4}$ axis. Now, here comes the question about the value for the closing point. One may think about the critical point

$$
\frac{\omega}{\gamma} F\left(x_{4}^{*}\right)=1
$$

that we can read off directly from the denominator. This point, however, corresponds to $\dot{x}_{4}=0$ which cannot be allowed in the reflection picture. But we know, that if the instanton is reflected at the pole of the weak rapid field, the path has to be closed there as well. So, in case of reflection, means if the latter field assists the vacuum decay, we have to set

$$
x_{4}^{\mathrm{c}} \stackrel{!}{=} x_{4}^{\mathrm{p}} \text {. }
$$

Otherwise, if we allow

$$
x_{4}^{\mathrm{c}}=x_{4}^{*},
$$

the instanton path will be closed before it can be reflected. This would simply mean that there will be no resizing due to reflections. This behaviour is schematically illustrated in Fig. 2. In the left panel we have the reflection picture where $x_{4}^{\mathrm{c}}=x_{4}^{\mathrm{p}}$. However, in the right panel we find the case for which the instanton is closed before reaching the pole, i.e. $x_{4}^{\mathrm{c}}<x_{4}^{\mathrm{p}}$. Thus, there will be no assistance due to the weak field. Its contribution will be
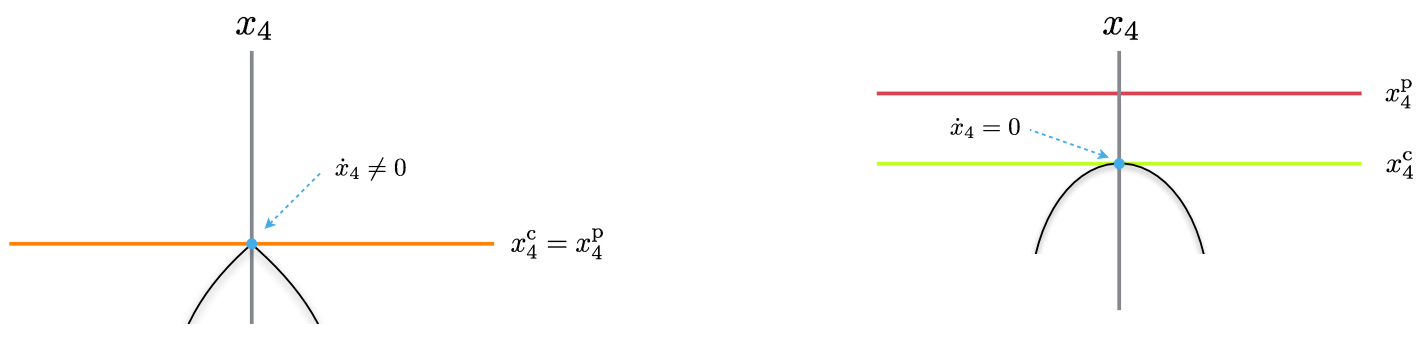

Figure 2. Comparison of dynamically assisted (left) and usual Schwinger mechanism (right) in temporally inhomogeneous electric background. The former is characterised by the additional instanton reflection due to the superposition with a weak rapid field. 
negligible and vacuum pair production is mainly driven by the strong field alone.

The reflection condition (5.10) from above shows that the profile of the weak highfrequent field, which basically determines the position of the pole $x_{4}^{\mathrm{p}}$, is crucial for the assisted vacuum decay. Moreover, the profile of the strong slow field, i.e. form of $f$, respectively, that determines the form of the integrand in (5.8), has substantial effect on the pair production rate. Only the interplay between both contributions will lead to a drastic enhancement of the rate.

However, for the latter we still need to compute the value of the modified kinematic invariant $a$ which determines, apart from its role in the previous expressions, the value of the kinetic term $\mathcal{W}_{0 \text {,kin }}=m a$, see (2.5) and (2.10), in the stationary worldline action $\mathcal{W}_{0}$. In fact, we will see that demanding the closing point $x_{4}^{\mathrm{c}}$ to be placed in $x_{4}^{\mathrm{p}}$, cf. (5.10), will lead to substantial modifications of the invariant $a$. These modifications will then change the shape and size of the instanton path, respectively. To begin with, let us first rewrite the following integration measure

$$
d u=d u \frac{d x_{4}}{d x_{4}}=\frac{d x_{4}}{\dot{x}_{4}} .
$$

Subsequently, in case of reflection, we can write the following integral relation

$$
1=\int_{0}^{1} d u=4 \int_{0}^{x_{4}^{\mathrm{p}}} \frac{d x_{4}}{\dot{x}_{4}}
$$

where we have accordingly set for the upper integration limit $x_{4}^{\mathrm{c}}=x_{4}^{\mathrm{p}}$. From this equality we can determine the kinematic invariant after inserting $\dot{x}_{4}$ from (5.4), that gives us the remaining relation

$$
a=4 \int_{0}^{x_{4}^{\mathrm{p}}} d x_{4} \frac{1}{\sqrt{1-\left(\frac{\omega}{\gamma} F\left(x_{4}\right)\right)^{2}}} .
$$

Altogether, combining the results (5.8) and (5.14), we find the following expression for the stationary worldline action

$$
\mathcal{W}_{0}=4 m \int_{0}^{x_{4}^{\mathrm{p}}} d x_{4} \sqrt{1-\left(\frac{\omega}{\gamma} F\left(x_{4}\right)\right)^{2}}
$$

\section{$6 \quad$ Fields with poles}

Let us illustrate the previous modifications in the reflection picture with some explicit examples. For this, we assume the strong field $\propto E$ to be static. Therefore we set

$$
f(t)=1, \quad F\left(x_{4}\right)=x_{4},
$$

since for larger frequencies, i.e. $\gamma \gg 1$, the effect of dynamical assistance vanishes if the weak field inhomogeneity remains as relatively small. This is due to a threshold value for the inhomogeneity which is characteristic for this mechanism $[6,11]$. Thus, in such a case 
the strong field alone will be sufficient to induce the enhancement. This corresponds to the usual situation in temporally inhomogeneous electric fields. This aspect is discussed in App. C. Due to the simple form of $F$, the integral in (5.14) can now be solved analytically as

$$
a=4 \int_{0}^{x_{4}^{\mathrm{p}}} d x_{4} \frac{1}{\sqrt{1-\left(\frac{\omega}{\gamma} x_{4}\right)^{2}}}=\left.4 \frac{\gamma}{\omega} \arcsin \left(\frac{\omega}{\gamma} x_{4}\right)\right|_{x_{4}=0} ^{x_{4}^{\mathrm{p}}} .
$$

Hence, we obtain the following kinematic invariant

$$
a=4 \frac{\gamma}{\omega} \arcsin \left(\frac{\omega}{\gamma} x_{4}^{\mathrm{p}}\right)
$$

depending on a general pole $x_{4}^{\mathrm{p}}$ that we have not specified yet. The latter expression shows that there will be some critical value for $\tilde{\gamma}$ depending on the exact value of $x_{4}^{\mathrm{p}}$, since $\frac{\omega x_{4}^{\mathrm{P}}}{\gamma}=\frac{\tilde{\omega} \mathrm{x}_{4}^{\mathrm{P}}}{\tilde{\gamma}} \leq 1$, which is the mentioned characteristic threshold in the assisted mechanism. So, the instanton path in the right half plane, i.e. $u \in[-1 / 4,1 / 4]$, see $(2.3)$, is simply an arch-like curve. With this the LMA condition (2.6) becomes

$$
\frac{m}{\omega} 4 \gamma \arcsin \left(\frac{\omega}{\gamma} x_{4}^{\mathrm{p}}\right) \gg 1 \text {. }
$$

After the integration of (2.12) and inserting the modified invariant (6.3), we obtain the following instanton solution

$$
\begin{aligned}
& x_{4}(u)=\frac{m}{e E} \sin \left(4 u \arcsin \left(\frac{\omega x_{4}^{\mathrm{p}}}{\gamma}\right)\right), \\
& x_{3}(u)=\frac{m}{e E} \cos \left(4 u \arcsin \left(\frac{\omega x_{4}^{\mathrm{p}}}{\gamma}\right)\right)-\mathcal{C} .
\end{aligned}
$$

The closed path is realised by the integration constant

$$
\mathcal{C}=x_{3}(u= \pm 1 / 4)=\frac{m}{e E} \cos \left(\arcsin \left(\frac{\omega x_{4}^{\mathrm{p}}}{\gamma}\right)\right) .
$$

It shifts the instanton along the $\hat{x}_{3}$ axis to guarantee the condition $x_{3}(u= \pm 1 / 4)=0$. Evaluating the stationary worldline action with (5.15) gives

$$
\begin{aligned}
\mathcal{W}_{0} & =4 m \int_{0}^{x_{4}^{\mathrm{p}}} d x_{4} \sqrt{1-\left(\omega x_{4} / \gamma\right)^{2}} \\
& =\frac{2 m}{\omega}\left(x_{4}^{\mathrm{p}} \omega \sqrt{1-\frac{x_{4}^{\mathrm{p}^{2}} \omega^{2}}{\gamma^{2}}}+\gamma \arcsin \left(\frac{x_{4}^{\mathrm{p}} \omega}{\gamma}\right)\right) .
\end{aligned}
$$

\section{Sauter}

To begin with, let us assume the weak field to be the in the literature well studied Sauter field, i.e.

$$
g(t)=\operatorname{sech}^{2}(\tilde{\omega} t), \quad G\left(x_{4}\right)=\frac{\tan \left(\tilde{\omega} x_{4}\right)}{\tilde{\omega}} .
$$



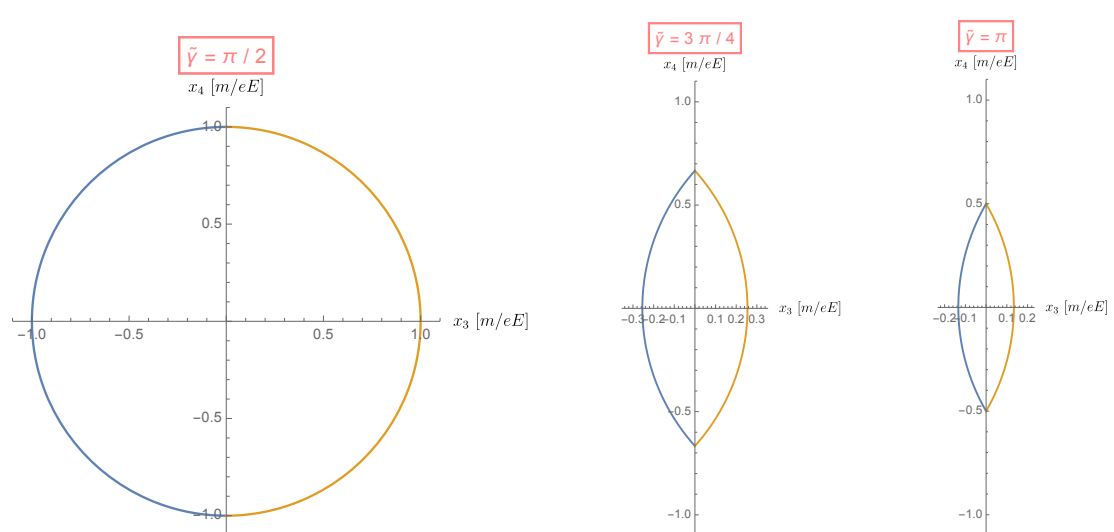

Figure 3. Instanton path for an electric field as superposition of a strong static and weak Sauter field.

The pole structure for this field is of multitype. However, the (first) relevant pole for $g\left(x_{4}\right)=\sec ^{2}\left(\tilde{\omega} x_{4}\right)$ is located in

$$
x_{4}^{\mathrm{p}}=\frac{\pi}{2 \tilde{\omega}}
$$

which leads to the following invariant

$$
a=4 \frac{m}{e E} \arcsin \left(\frac{\pi}{2 \tilde{\gamma}}\right)
$$

The above result indicates that the combined Keldysh parameter has to satisfy $\tilde{\gamma}>\pi / 2$, otherwise we would find $a \in \mathbb{C}$ which is forbidden, means no closed instanton solution can exist. Below this critical value,

$$
\tilde{\gamma}^{\text {crit }}=\frac{\pi}{2}
$$

there will be no drastic effect of the weak field and we are left with the static field case. This is the known result in [6] obtained for a strong Sauter field in the limit $\omega \ll \tilde{\omega}$. In this limit, the strong field can be assumed as locally static, as it is seen by the weak rapid field. It is clear that this can only be expected for the case of large frequency differences. As soon as the lower frequency approaches larger values, the inhomogeneity of the strong field will dominate below the critical value $\tilde{\gamma}^{\text {crit }}$. This situation will be discussed in Sec. 8 . Above the critical threshold, it will be again the weak high-frequent field that enhances the vacuum decay via the assisted mechanism.

Coming back to the present example, inserting the pole $x_{4}^{\mathrm{p}}$ into the modified solutions (6.5), we plot the instanton path for different frequencies $\tilde{\omega}$ as shown in Fig. 3. As one can observe, the size of the instanton decreases with larger combined Keldysh parameter $\tilde{\gamma}$. This shrinking will then increase the rate $\mathcal{R} \sim \exp \left(-\mathcal{W}_{0}\right)$, since the stationary action decreases as soon as the size of the instanton is reduced. Such lense shaped instantons also apply if the strong field is a spatially inhomogeneous Sauter field [77]. After inserting the pole into the solution (6.7), the stationary worldline action for the assisted process reads

$$
\mathcal{W}_{0} \approx \frac{m^{2}}{e E}\left(\frac{\pi}{2 \tilde{\gamma}^{2}} \sqrt{4 \tilde{\gamma}^{2}-\pi^{2}}+2 \arcsin \left(\frac{\pi}{2 \tilde{\gamma}}\right)\right) .
$$


This is again the literature result [6]. Alternatively, we would get this result by plugging the instanton solution from above into (5.15) and integrating over $u \in[0,1 / 4]$.

\section{Lorentzian}
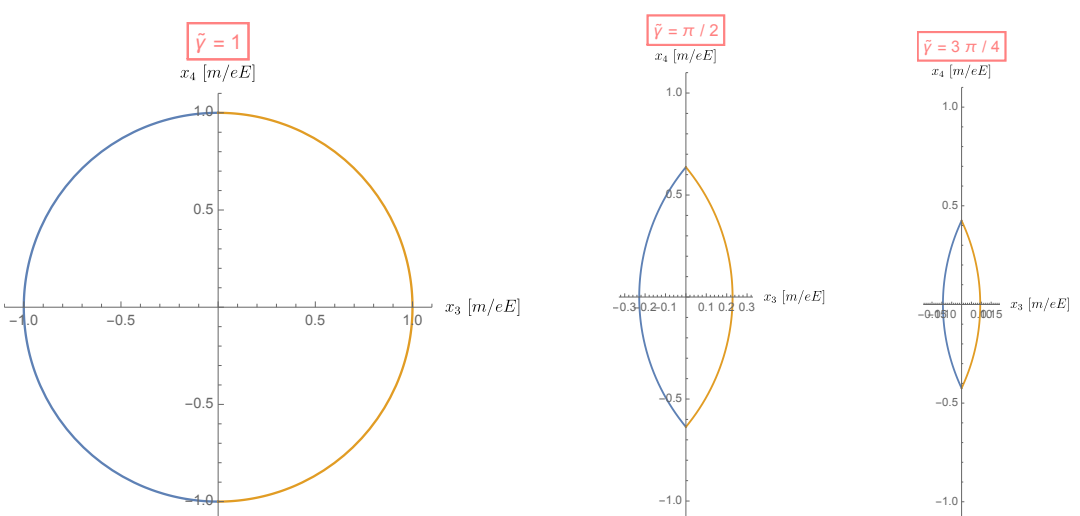

Figure 4. Instanton path for an electric field as superposition of a strong static and weak Lorentzian field.

Another example we can compute in an analogous way is possible for a Lorentzian weak field. This field profile can be described by

$$
g(t)=\frac{1}{\left(1+(\tilde{\omega} t)^{2}\right)^{3 / 2}}, \quad G\left(x_{4}\right)=\frac{1}{\tilde{\omega}} \frac{\tilde{\omega} x_{4}}{\sqrt{1-\left(\tilde{\omega} x_{4}\right)^{2}}} .
$$

It belongs to the type of fields which have a bell-shaped profile like the previous Sauter field. The corresponding (analytic continued) function $g\left(x_{4}\right)=1 /\left(1-\left(\tilde{\omega} x_{4}\right)^{2}\right)^{3 / 2}$ has a distinct pole structure as well, which is identified as

$$
x_{4}^{\mathrm{p}}=\frac{1}{\tilde{\omega}} .
$$

Except the factor $\pi / 2$, it resembles the case before. Therefore, we expect similar results for the Lorentzian field. However, one should remark that not the visually indistinguishable bell-shaped profile is responsible for this similarity ${ }^{7}$, cf. e.g. [11]. This point will be discussed in detail in Sec. 7. The modified invariant for this Lorentzian field is

$$
a=4 \frac{m}{e E} \arcsin \left(\frac{1}{\tilde{\gamma}}\right)
$$

which obviously leads to a smaller critical combined Keldysh parameter

$$
\tilde{\gamma}^{\text {crit }}=1
$$

cf. e.g. [64]. Thus the instanton reflection happens already with $\tilde{\gamma}=1$. Consequently, the weak field will start to contribute much earlier compared to the Sauter field case. This

\footnotetext{
${ }^{7}$ It is the distinct pole structure of the field which is responsible for the expected similarity.
} 
behaviour is illustrated in Fig. 4. Inserting the pole into (6.7), we find the stationary worldline action [64]

$$
\mathcal{W}_{0} \approx \frac{m^{2}}{e E}\left(\frac{2}{\tilde{\gamma}^{2}} \sqrt{\tilde{\gamma}^{2}-1}+2 \arcsin \left(\frac{1}{\tilde{\gamma}}\right)\right)
$$

The comparison of $\mathcal{W}_{0}$ for both fields is shown in Fig. 5. The difference with respect to the criticality is clearly observable. Despite the relative difference between the curves,

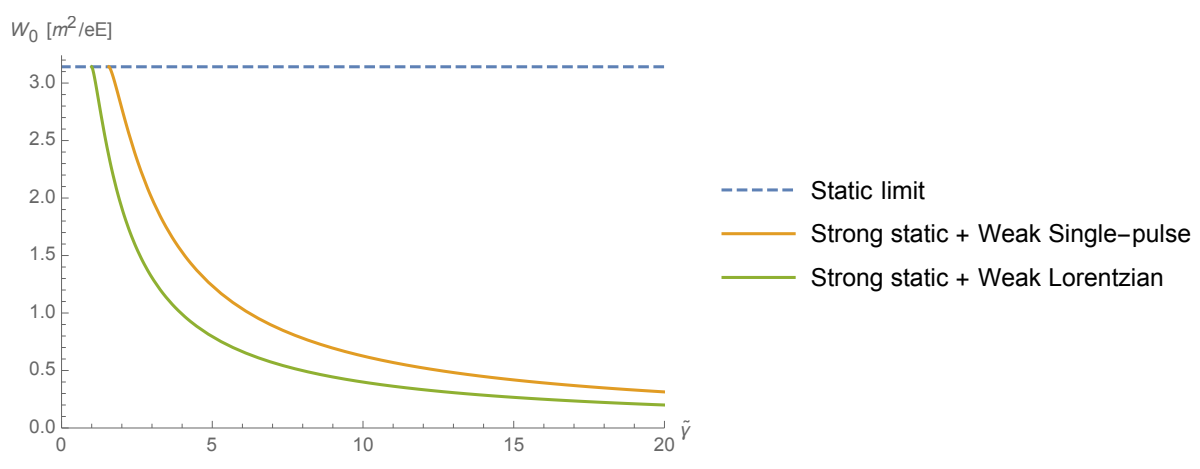

Figure 5. Stationary worldline action $\mathcal{W}_{0}\left[\frac{m^{2}}{e E}\right]$ for the electric field as superposition of a strong static and weak Sauter/Lorentzian (yellow/green curve) field. The horizontal blue line corresponds to the static field case with $\mathcal{W}_{0}\left[\frac{m^{2}}{e E}\right]=\pi$.

we observe that both behave similarly. Identical results will apply, if we increase the frequency of the Sauter field by a factor $\frac{\pi}{2}$. This we can already observe directly in Fig. 5 . For example, in case of the Lorentzian field the value $\approx 1.8$ is reached at $\tilde{\gamma} \approx 2$. The same result applies for the Sauter field at $\tilde{\gamma} \approx 3.2$. This is almost the mentioned factor $\frac{\pi}{2}$, the difference which results from the poles $x_{4}^{\mathrm{p}}$. In Sec. 7 we will discuss other examples with bell-shaped fields which behave differently from the one presented here. Those studies will support the assumption that the main difference is rather determined by the pole structure of the electric field, at least for sufficiently small $\epsilon$, which is basically the limit in which the effect of dynamical assistance sets in.

\section{Effects of sub-cycle structure}

The previous two examples have shown that for sufficiently small $\epsilon$ the assistance is mainly determined by the poles of the bell-shaped weak fields. This feature has been also confirmed by exact numerical computations. In the following we study the changes in case the field possesses some oscillatory sub-structure. This situation is reflected in laser setups where the field consists of a very short wave packet ${ }^{8}$. Hence, the question is, how such a subcycle structure will influence the rate? As an illustrative example, let us assume a simple

\footnotetext{
${ }^{8}$ Despite the fact that those are electromagnetic pulses, a pure electric field of this type is still a good approximation. It can be realised, for instance, through a collision of two counter-propagating laser pulses equal in their (linear) polarisation and intensity.
} 
oscillatory pulse described by

$$
g(t)=\frac{1-3(\tilde{\omega} t)^{2}-2(N \tilde{\omega} t)^{2}}{\left(1+(N \tilde{\omega} t)^{2}\right)^{5 / 2}}, \quad G\left(x_{4}\right)=\frac{1}{\tilde{\omega}} \frac{\tilde{\omega} x_{4}+\left(\tilde{\omega} x_{4}\right)^{3}}{\left(1-\left(N \tilde{\omega} x_{4}\right)^{2}\right)^{3 / 2}}, \quad N \geq 1 .
$$

Its comparison with the Lorentzian profile (6.13), which is the inverse of the demoniator, is depicted in Fig. 6 (top-left) for $N=1$. For this type of field we find analogously the generalised Lorentzian pole

$$
x_{4}^{\mathrm{p}}=\frac{1}{N \tilde{\omega}},
$$

which will be again responsible for the instanton reflection and hence the relevant quantity in the assisted mechanism. For sufficiently small $\epsilon$, for which the reflection at the infinitely high potential wall in $x_{4}^{\mathrm{p}}$ is the main mechanism behind the dynamical assistance, the subcycle structure would be irrelevant. It is the pole structure of the bell-shaped Lorentzian part that regulates the strength of the enhancement. This expectation is exactly confirmed in Fig. 6. In the top-right panel we have plotted again the stationary worldline action for the previously discussed Lorentzian field, but know comparing it with exact numerical computations for different $\epsilon$. For $\epsilon<0.01$ the analytical prediction and the numerical result are almost identical. Only for $\epsilon=0.01$ there appears a notable difference. Doing the same computation for the oscillatory pulse, (6.18), we identify a similar behaviour. Despite the fact, that for larger $\epsilon$ one observes a stronger deviation, which is completely plausible, since the total effective field strength becomes larger, we can again observe for small $\epsilon$ that our prediction agrees very well with the exact numerical computations. Hence, having obtained the exact reflection point turns out to be sufficient to predict the stationary action for $\epsilon \ll 1$. As we know, this is basically the attractive regime for the dynamical assistance. Note, that for the limit $\tilde{\gamma} \gg 1$, both curves converge, independent of the relative field strength ratio $\epsilon$.

The previous observation is very interesting, since usually pulsed fields, as shown in the present example, are described by an appropriate oscillatory function which is multiplied with some bell-shaped function, for example the Lorentzian field applied here. Usually those envelope functions have poles which are closer to the origin than the effective reflection points for (infinitely extended) oscillating fields, as for instance the sinusoidal field. The latter situation will be discussed in Sec. 7. Thus, it is exactly the latter pole originating from the envelope function, which will predominantly determine the reflection of the instanton. Note that a profile, which can model such a pulse field, may also exist without a distinct pole structure. An example is the Gaussian field, addressed in Sec. 7 as well.

We have discussed that, at least for $\epsilon \ll 1$, the assistance is dominantly determined by the pole structure of the bell-shaped envelope function that regulates the pulse width, and not the encased oscillatory sub-structure. However, one should note that those insights are derived via computing the (totally integrated) stationary worldline action $\mathcal{W}_{0}$. As soon as one resolves the momentum spectrum of the produced pairs, differences due to the inner sub-cycle structure of the pulse should be visible. Namely, the latter can be very decisive, basically in form of quantum interference effects highlighted in the phasespace of the produced pairs, cf. e.g. [8, 83-85, 89]. Moreover, note that those features 
will substantially change for fields which do not fulfil the symmetry properties we have assumed in the present paper.
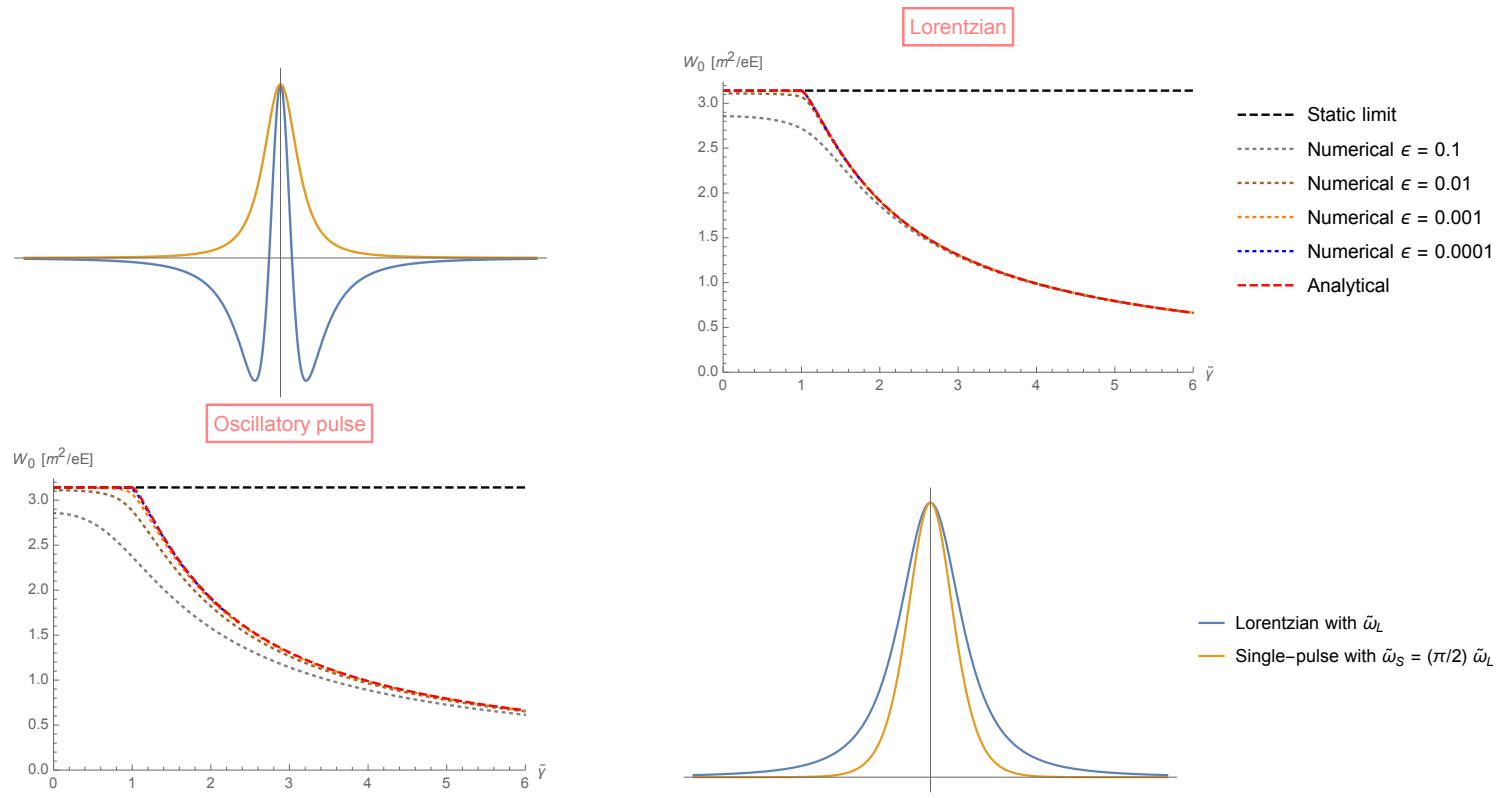

Figure 6. Analytically and numerically computed stationary worldline action for the oscillatory pulse (6.18) with $N=1$ (bottom-left) and Lorentzian (6.13) (top-right) profile. The values for $\epsilon$ are given in the plot legend. The function $g(t)$ for the oscillatory pulse (blue) and Lorentzian field (yellow) is plotted in the top-left panel. The comparison between the Sauter and Lorentzian field is depicted in the bottom-right panel where the frequency for the former one is multiplied by $\pi / 2$. This leads to the same reflection point $x_{4}^{\mathrm{p}}$, cf. (6.9) and (6.14). Even the profiles look different, the corresponding stationary actions $\mathcal{W}_{0}$ are identical for $\epsilon \lesssim 10^{-3}$.

Furthermore, the critical pole of the Lorentzian field is reached if we multiply the frequency of the Sauter field by a factor $\frac{\pi}{2}$. For a sufficiently small $\epsilon$, say $\epsilon=10^{-3}$, the rate is identical for both cases, which is also confirmed by numerical computations. However, looking on the electric field profiles for these frequencies, one can observe a clear difference, cf. Fig. 6 (bottom-right). This is similar to the previous situation with the oscillatory pulse. The field profiles look completely different. Despite this, for $\epsilon=10^{-3}$ we find almost identical results if we apply the reflection point $x_{4}^{\mathrm{p}}=\frac{1}{\tilde{\omega}}$. This clarifies, that for a sufficiently weak field, the assistance is mainly determined by the latter's pole structure. Although the field profiles may look very dissimilar, those will lead to same results if the poles are identical.

A second important aspect regarding the Lorentzian field (6.13) is as follows. Let us apply a field with a more general profile described by

$$
g(t)=\frac{1}{\left(1+(\tilde{\omega} t)^{2}\right)^{d / 2}}
$$

where $d \in \mathbb{N}$. Apparently, we will obtain the same pole as before, which is the inverse of the field frequency $\tilde{\omega}$. The reason for this universal pole is a special feature of this type 
of field profile. Namely, it is completely equivalent if one varies $d$ in the exponent of the denominator or the frequency $\tilde{\omega}$. For any $d$ one always can find a corresponding $\tilde{\omega}$ that leads exactly to the same field profile. Hence, for fields of the latter type it is sufficient to find the frequency-dependent pole $x_{4}^{\mathrm{p}}$, which simultaneously covers all possible configurations with an arbitrary exponent $\frac{d}{2}$.

\section{$7 \quad$ Fields without poles}
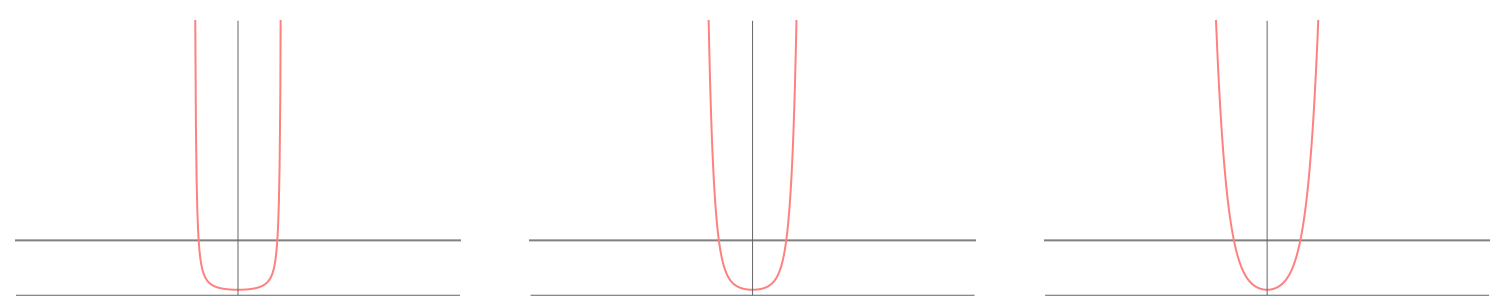

Figure 7. Intersection points between the strong and weak field as reflecting mirrors: The functions $f\left(x_{4}\right)$ (gray line) and $g\left(x_{4}\right)$ (pink curves) are plotted schematically versus $x_{4}$. After rotation in the complex plane the strong static field becomes again static (gray line). The inhomogeneous weak field, however, becomes a positive monotonic function (pink curves). The weak field is assumed to be Lorentzian (left), Gaussian (center) and sinusoidal (right). Note that, in contrast to the Lorentzian field, the last two have not a distinct pole structure.

So far we have considered fields which have a distinct pole structure in Euclidean spacetime. However, the assisted mechanism operates more general, i.e. also in other inhomogeneous weak fields with completely different profile $[7-11,14,90]$. A well considered candidate is the sinusoidal field. This field is extended to infinity and has no distinct pole structure as in the cases before. However, we discussed, that the reflection of the instanton by an infinite potential wall turned out to be the main mechanism behind the process of dynamically assisted vacuum pair production. Hence, we will look again for such reflecting mirrors in order to employ the above formalism.

Since previous geometrical arguments seem to be less intuitive, we consider a more general concept: in the limit $\tilde{\omega} \gg \omega$, the function $g\left(x_{4}\right)$ will be curving much stronger than the slower function $f\left(x_{4}\right)$. For sufficiently large frequencies $\tilde{\omega}$, such a bending results in a potential-wall-like structure confining some region of the strong static curve. This situation is sketched in Fig. 7. The left panel shows the case for the Lorentzian field. The infinite wall is formed in the field poles $x_{4}^{\mathrm{p}}$. In the remaining two other cases, a Gaussian (center) and sinusoidal (right) field, we find similar structures. Although, those fields do not have poles, their superposition with the static line form some effective reflectors. Those are located around the intersection points between the strong and weak field. Therefore, we expect, at least for a sufficiently large frequency $\tilde{\omega}$ or combined Keldysh parameter $\tilde{\gamma}$, respectively, that the intersection points, which we denote in the following by $x_{4}^{\mathrm{i}}$, will play a similar role as the poles for the Lorentzian field in the left panel do. Hence, we may assume for the effective reflection point

$$
x_{4}^{\mathrm{p}} \approx x_{4}^{\mathrm{i}} .
$$


The reflection applies where both curves intersect with each other. Thus, we have to solve the equation for the derivatives

$$
F^{\prime}\left(x_{4}^{\mathrm{i}}\right) \stackrel{!}{=} \epsilon G^{\prime}\left(x_{4}^{\mathrm{i}}\right)
$$

which follows alternatively from (4.4).
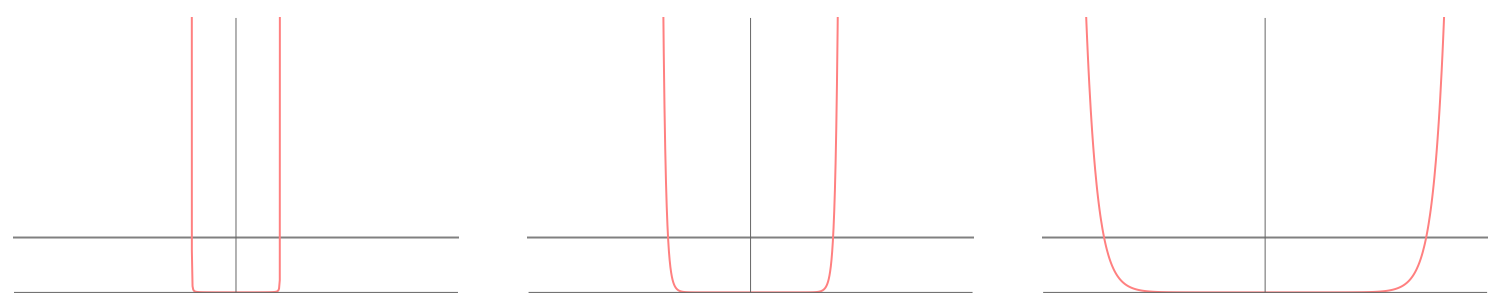

Figure 8. Similar plot as in Fig. 7. The strength of the weak field is decreased by 5 orders of magnitude compared to the latter plots. The poles for the Lorentzian field (left) do not change. However, the position of the intersection points for the Gaussian (center) and sinusoidal (right) field depend on the parameter $\epsilon$.

From the latter condition it is apparent that the effective reflection point will depend on the strength parameter $\epsilon$ as well. This is in concordance with recent studies, cf. e.g. [11]. Such an $\epsilon$ dependence will also apply for the quantum fluctuation prefactor, as recently observed in numerical investigations [64]. Further studies regarding the fluctuation prefactor will be addressed elsewhere [65]. The $\epsilon$ dependence is schematically shown in Fig. 8. For fields with true poles, as the Lorentzian field (left panel), the position of the pole does not depend on the field strength, i.e. $\epsilon$. However, for the remaining two other fields one can observe a huge difference in comparison to Fig. 7. The strength of the weak field is decreased by five orders of magnitude relatively to the previous plot. One may expect that the prescribed procedure will be more accurate as soon as the parameter $\epsilon$ becomes small, since this leads to a very fast increase of the weak field curve in the intersection points. This is basically the same behaviour, one would find in the vicinity of a true pole, cf. left plot in Fig. 8. Moreover, for the two poleless fields we expect that the critical point, in which the weak field starts to contribute considerably, drifts more towards the intersection point, that is initially assumed to be the effective reflector, as soon $\epsilon$ is decreased. This is indeed an essential property for such poleless fields. More details regarding this interesting issue will be discussed in the following.

Before we proceed with the analytic computations, let us emphasise the following aspect. Comparing the curves for the Gaussian and sinusoidal field, we observe that the slope of the curve for the former field seems to be much stronger around the intersection points. Due to this difference, we expect more accurate predictions in this simplified approach for the Gaussian field. In addition, there will be still some contribution of the weak field, particularly, right after the intersection points, which should be relevant. However, this detail will not be taken into account in our simplified picture with the intersection points as effective reflectors. 


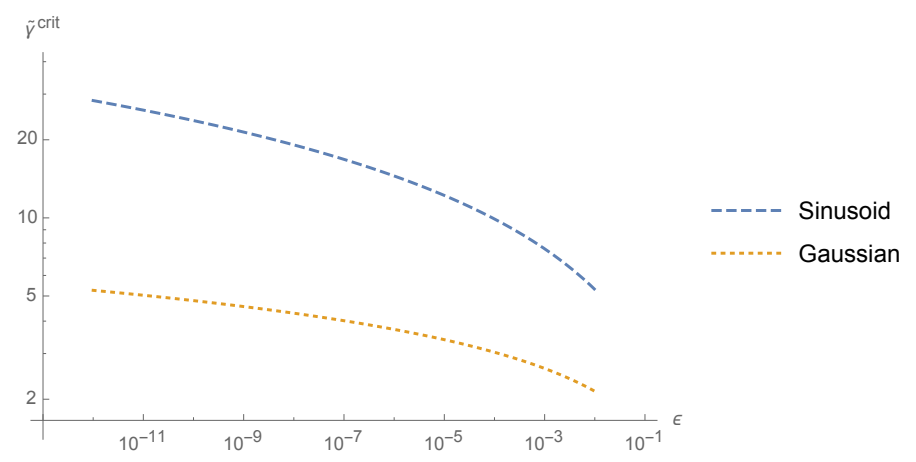

Figure 9. Critical combined Keldysh parameter $\tilde{\gamma}^{\text {crit }}$ for the weak sinusoidal and Gaussian field, superimposed with a strong static field, is plotted versus $\epsilon$, where $\tilde{\gamma}^{\text {crit }}$ is evaluated assuming the critical point to be $x_{4}^{\mathrm{i}}$.

\section{Sinusoid}

We begin with a sinusoidal shaped weak field that is characterised by

$$
g(t)=\cos (\tilde{\omega} t), \quad G\left(x_{4}\right)=\frac{\sinh \left(\tilde{\omega} x_{4}\right)}{\tilde{\omega}} .
$$

Inserting the corresponding derivatives $F^{\prime}, G^{\prime}$ into (7.2) leads to the following intersection point

$$
x_{4}^{\mathrm{i}}=\frac{\operatorname{arccosh}(1 / \epsilon)}{\tilde{\omega}} .
$$

Using the equality condition in (7.1), we can directly obtain the modified kinematic invariant applying (6.3) and (5.1),

$$
a \approx 4 \frac{\gamma}{\omega} \arcsin \left(\frac{1}{\tilde{\gamma}} \operatorname{arccosh}(1 / \epsilon)\right) .
$$

For the critical combined Keldysh parameter we find from the latter expression (or alternatively by solving (8.5))

$$
\tilde{\gamma}^{\text {crit }}=\operatorname{arccosh}(1 / \epsilon) \approx \ln (2 / \epsilon) \approx|\ln (\epsilon)|
$$

Note that this estimation already agrees with the approximate WKB (Wentzel-KramersBrillouin) approximation for the case $p=0$ found in [11]. The above result is depicted in Fig. 9. For the approximations in the latter expression we have assumed the condition $\epsilon \ll 1$. This is the relevant regime in the present reflection picture.

Subsequently, applying the general result from (6.5), we obtain the approximate instanton path described by

$$
\begin{aligned}
& x_{4}(u) \approx \frac{m}{e E} \sin \left(4 u \arcsin \left(\frac{\operatorname{arccosh}(1 / \epsilon)}{\tilde{\gamma}}\right)\right), \\
& x_{3}(u) \approx \frac{m}{e E} \cos \left(4 u \arcsin \left(\frac{\operatorname{arccosh}(1 / \epsilon)}{\tilde{\gamma}}\right)\right)-\mathcal{C}
\end{aligned}
$$


with $\mathcal{C}=x_{3}(u= \pm 1 / 4)$. Inserting (7.4) into (6.7), the corresponding stationary worldline action evaluated on the instanton (7.7), which is assumed to be reflected in the intersection point $x_{4}^{\mathrm{i}}$, reads

$$
\mathcal{W}_{0} \approx \frac{m^{2}}{e E}\left(\frac{2 \operatorname{arccosh}(1 / \epsilon)}{\tilde{\gamma}^{2}} \sqrt{\tilde{\gamma}^{2}-\operatorname{arccosh}^{2}(1 / \epsilon)}+2 \arcsin \left(\frac{\operatorname{arccosh}(1 / \epsilon)}{\tilde{\gamma}}\right)\right) .
$$

The resulting plots for the instanton (7.7) as well as the stationary worldline action (7.8) are attached in App. D. We observe that, although the analytical prediction follows the trend of the exact numerical curve, they considerably differ from each other.

As we commented in the introductory part, such a distinction was actually expected because of the instanton reflection, which we have assumed precisely in the intersection point. We argued that there will be some region of the weak field after the intersection point, which will surely have a non-negligible contribution to the pair production rate. This is not taken into account when we assume $x_{4}^{\mathrm{p}}=x_{4}^{\mathrm{i}}$. Before we present the improved effective reflection point, let us first introduce a second example which is expected to be sharing similar features under the latter assumption.

\section{Gaussian}

The second example without a distinct pole structure is the Gaussian field characterised by

$$
g(t)=\exp \left(-(\tilde{\omega} t)^{2}\right), \quad G\left(x_{4}\right)=\frac{\sqrt{\pi} \operatorname{erfi}\left(\tilde{\omega} x_{4}\right)}{2 \tilde{\omega}} .
$$

The intersection point in this case is given by

$$
x_{4}^{\mathrm{i}}=\frac{\sqrt{\ln (1 / \epsilon)}}{\tilde{\omega}} .
$$

Proceeding similarly, we get the approximate invariant from (6.3)

$$
a \approx 4 \frac{\gamma}{\omega} \arcsin \left(\frac{1}{\tilde{\gamma}} \sqrt{\ln (1 / \epsilon)}\right)
$$

which leads to the critical Keldysh parameter

$$
\tilde{\gamma}^{\text {crit }}=\sqrt{\ln (1 / \epsilon)}=\sqrt{|\ln (\epsilon)|} .
$$

As in the previous case, this result is again the approximate estimation within the WKB approach [11]. The critical value is plotted in Fig. 9. It is notable that for small $\epsilon$ the trend for the Gaussian field turns out to be more peak-like than for the sinusoidal field. Therefore, for the Gaussian field, we expect that analytical predictions will lead to more accurate results for sufficiently small $\epsilon$. Note that as soon as $\epsilon$ decreases, the true critical point (in which the weak field contribution starts to be substantial) will approach the intersection point. Inserting the intersection point again in the instanton solution (6.5), 
we continue with

$$
\begin{aligned}
& x_{4}(u) \approx \frac{m}{e E} \sin \left(4 u \arcsin \left(\frac{\sqrt{\ln (1 / \epsilon)}}{\tilde{\gamma}}\right)\right), \\
& x_{3}(u) \approx \frac{m}{e E} \cos \left(4 u \arcsin \left(\frac{\sqrt{\ln (1 / \epsilon)}}{\tilde{\gamma}}\right)\right)-\mathcal{C},
\end{aligned}
$$

where the constant $\mathcal{C}=x_{3}(u= \pm 1 / 4)$ plays the same role as before. We can again plug (7.10) into (6.7), which leads to the corresponding stationary action

$$
\mathcal{W}_{0} \approx \frac{m^{2}}{e E}\left(\frac{2 \sqrt{\ln (1 / \epsilon)}}{\tilde{\gamma}^{2}} \sqrt{\tilde{\gamma}^{2}-\ln (1 / \epsilon)}+2 \arcsin \left(\frac{\sqrt{\ln (1 / \epsilon)}}{\tilde{\gamma}}\right)\right) .
$$

For completeness, the plots for (7.13) and (7.14) have been attached to App. D, cf. Figs. 23 and 24. From those, we still find a clear deviation for large $\tilde{\gamma}$ compared to the numerics. However, this distinction is significantly smaller than in the case before. This agrees with our expectation based on the observation in Fig. 9.

\section{Improving the mirrors}

As we have explicitly illustrated in the previous two examples, reflecting the instanton in the intersection points $x_{4}^{\mathrm{i}}$ can mimic the general trend of the reduced stationary action or enhanced pair production rate, respectively. However, we have observed that the predictions still differ considerably from the exact numerical computations.

In the following, we show how to improve the effective reflection points considerably. Furthermore, these improvements confirm the generalization of the reflection picture for poleless fields. Some region after the intersection points has to be taken into account for an accurate prediction for the stationary action $\mathcal{W}_{0}$. Hence, we write for the improved effective reflection point

$$
x_{4}^{\mathrm{p}} \rightarrow x_{4}^{\mathrm{i}}+\frac{\delta}{\tilde{\omega}},
$$

where $\delta / \tilde{\omega}$ stands for the corresponding correction with the displacement parameter $\delta$. Such a geometrical uncertainty regarding the exact position of the effective reflection point, turns out to be the characteristic attribute for fields without a distinct pole structure. For the type of fields with true poles, such uncertainties do not occur.

Once we determine the parameter $\delta$, the relevant combined Keldysh parameter $\tilde{\gamma}$ in the final expressions has to be modified, since the critical parameter $\tilde{\gamma}^{\text {crit }}$ will still be determined by the intersection point ${ }^{9}$.

However, one should keep in mind that the latter is not exact with respect to the true critical threshold. The true value will be smaller. The critical point, which determines the

\footnotetext{
${ }^{9}$ This is in general characteristic for fields without poles. There is always a difference between the effective reflection point and the critical point in which the weak field begins to contribute. For fields with a distinct pole structure this disagreeance vanishes, at least in the highly weak limit, i.e. $\epsilon \ll 1$, which is the attractive and relevant regime for the assisted mechanism.
} 
true critical threshold for fields without poles, is rather of the form

$$
(1-\Delta) x_{4}^{\mathrm{i}}
$$

which leads to the true critical Keldysh parameter

$$
(1-\Delta) \tilde{\gamma}^{\text {crit }}
$$

The latter corrections can be determined by perturbation around the intersection point we have introduced before, cf. App. B. Note that those will provide a correction to the previous results (7.6) and (7.12), respectively, which are in agreement with estimations obtained in WKB approximation with $p=0$ [11].

However, applying the previous value $\tilde{\gamma}^{\text {crit }}$, will only influence the region for $\tilde{\gamma}$ around the critical value. For large combined Keldysh parameter $\tilde{\gamma}>\tilde{\gamma}^{\text {crit }}$, which is the relevant limit, we expect no deviations from numerical simulations. Therefore, in this limit, $x_{4}^{\mathrm{i}}$ taken as the critical point, is assumed to be sufficient for a good approximation and we do the following ansatz for improving the effective reflection point

$$
\begin{aligned}
x_{4}^{\mathrm{p}} & \rightarrow x_{4}^{\mathrm{i}}+\frac{\delta}{\tilde{\omega}}, \\
\tilde{\gamma} & \rightarrow \tilde{\gamma}+\delta .
\end{aligned}
$$

Such a shifted Keldysh parameter signals already that the impact of the weak rapid field on the rate $\mathcal{R}$ substantially differs for profiles with and without poles. For the former case the $\tilde{\gamma}$ dependence is determined only by the pole, whereas in the latter case there will be an additional dependence on $\epsilon$.

Further relevant aspects regarding the basic assumptions and derivations of the improvements can be read in more detail in App. A (regarding $\delta$ ) and B (regarding $\Delta$ ), respectively.

\section{Sinusoid: improved}

Let us begin with the sinusoidal field. According to the modifications in (7.18), we need to compute $\delta$. Applying the inverse function

$$
G^{-1}\left(x_{4}\right)=\frac{\operatorname{arcsinh}\left(\tilde{\omega} x_{4}\right)}{\tilde{\omega}}
$$

to (A.7), we obtain the following result

$$
\delta=\operatorname{arcsinh}\left(\frac{\tilde{\gamma}}{\epsilon}\right)-\operatorname{arccosh}\left(\frac{1}{\epsilon}\right) .
$$

Inserting the corresponding replacements afterwards,

$$
\begin{aligned}
\operatorname{arccosh}(1 / \epsilon) & \rightarrow \operatorname{arccosh}(1 / \epsilon)+\delta, \\
\tilde{\gamma} & \rightarrow \tilde{\gamma}+\delta,
\end{aligned}
$$


into (7.5), (7.7) and (7.8), we obtain the improved invariant $a$, instanton path and stationary worldline action $\mathcal{W}_{0}$, respectively. The latter written out explicitly reads

$$
\begin{aligned}
\mathcal{W}_{0} & \approx \frac{m^{2}}{e E}\left(\frac{2(\operatorname{arccosh}(1 / \epsilon)+\delta)}{(\tilde{\gamma}+\delta)^{2}} \sqrt{(\tilde{\gamma}+\delta)^{2}-(\operatorname{arccosh}(1 / \epsilon)+\delta)^{2}}\right. \\
& \left.+2 \arcsin \left(\frac{\operatorname{arccosh}(1 / \epsilon)+\delta}{\tilde{\gamma}+\delta}\right)\right) .
\end{aligned}
$$

The improved action (7.23) is plotted in Fig. 10 for $\epsilon=\left\{10^{-3}, 10^{-6}\right\}$, clearly showing a substantial improvement of the approximate analytical result in contrast to the predictions in Fig. 23. As we expected, despite the region around the critical combined Keldysh parameter (vertical dashed red line), the analytical curve is in good agreement with the exact numerical curve.
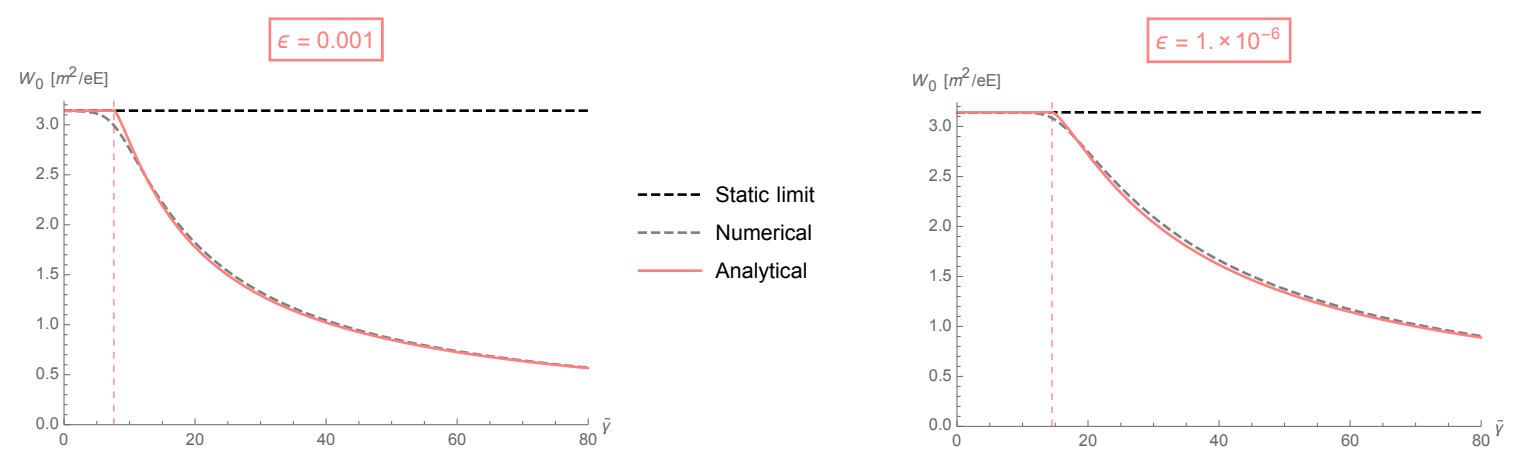

Figure 10. Stationary worldline action $\mathcal{W}_{0}$ for the sinusoidal weak field (7.3). The analytical prediction (7.23) is compared with the exact numerical result. The ratio between the strong and weak field strengths is set to $\epsilon=\left\{10^{-3}, 10^{-6}\right\}$ (left,right). The vertical dashed red lines are placed at $\tilde{\gamma}=\tilde{\gamma}^{\text {crit }}$. The improved effective reflection point $x_{4}^{\mathrm{p}}=x_{4}^{\mathrm{i}}+\delta / \tilde{\omega}$ has been applied.

\section{Gaussian: improved}

We can follow the same procedure as before to improve our analytical predictions for the Gaussian field (7.9). The displacement parameter $\delta$ is smaller compared to the case with the sinusoidal field (7.3). This is simply due to the reason that the slope of the function $g\left(x_{4}\right)$ in the vicinity of the intersection point is much stronger, e.g. cf. Fig. 8.

Apart from this expectation, there is another difference for the present setup: the function $G\left(x_{4}\right)$ is the imaginary error function for which the inverse is difficult to express algebraically. However, cf. the discussion in App. A, we can first apply (A.16) and Taylor expand the nonlinearity in $\xi$ introduced via the condition (A.13), since $\xi<1$. Proceeding in that way, we obtain the following result in leading order

$$
\xi \approx \frac{1}{\sqrt{2}} \sqrt{\frac{2}{\ln \left(\frac{1}{\epsilon}\right)}-\frac{\sqrt{\pi} \epsilon \operatorname{erfi}\left(\sqrt{\ln \left(\frac{1}{\epsilon}\right)}\right)}{\ln ^{\frac{3}{2}}\left(\frac{1}{\epsilon}\right)}},
$$

depending only on $\epsilon$, which is implicitly required via (A.12) and (A.13). 
Finally, using the critical Keldysh parameter (7.12) we can determine the displacement parameter

$$
\delta=\xi \sqrt{\ln (1 / \epsilon)}
$$

Inserting the replacements

$$
\begin{aligned}
\sqrt{\ln (1 / \epsilon)} & \rightarrow \sqrt{\ln (1 / \epsilon)}+\delta, \\
\tilde{\gamma} & \rightarrow \tilde{\gamma}+\delta
\end{aligned}
$$

into (7.11), (7.13) and (7.14), we obtain for the Gaussian field the improved invariant $a$, instanton path and stationary worldline action $\mathcal{W}_{0}$, respectively. The action reads explicitly

$$
\begin{aligned}
\mathcal{W}_{0} & \approx \frac{m^{2}}{e E}\left(\frac{2(\sqrt{\ln (1 / \epsilon)}+\delta)}{(\tilde{\gamma}+\delta)^{2}} \sqrt{(\tilde{\gamma}+\delta)^{2}-(\sqrt{\ln (1 / \epsilon)}+\delta)^{2}}\right. \\
& \left.+2 \arcsin \left(\frac{\sqrt{\ln (1 / \epsilon)}+\delta}{\tilde{\gamma}+\delta}\right)\right) .
\end{aligned}
$$

The comparison between the analytically predicted action $\mathcal{W}_{0}(7.28)$ and its exact numer-
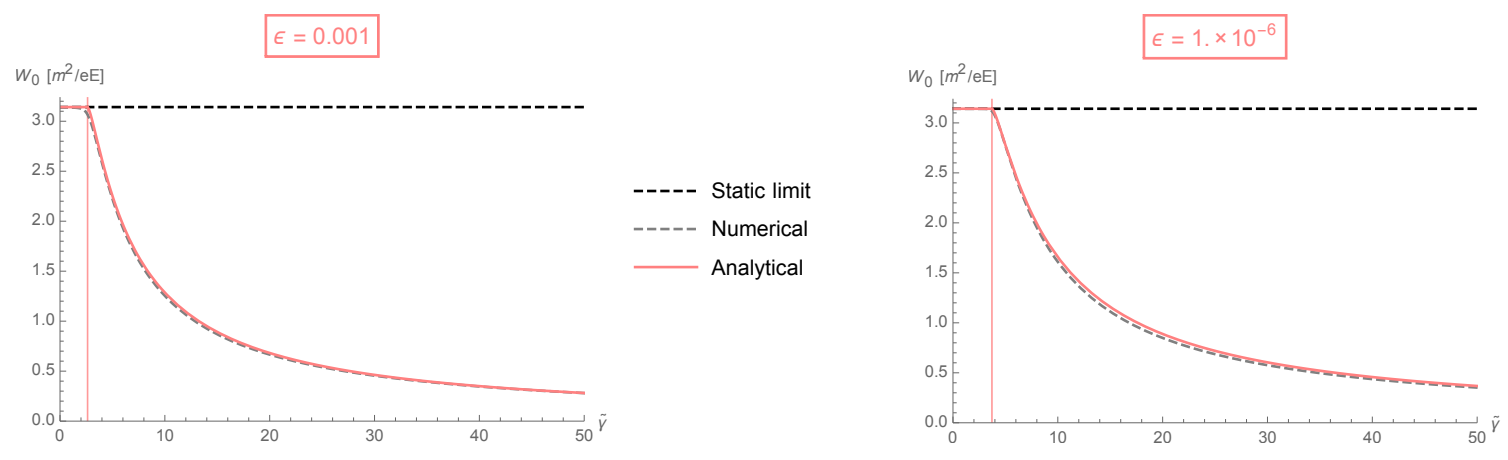

Figure 11. $\mathcal{W}_{0}$ for the weak Gaussian field (7.9). The analytical prediction (7.28) is compared with the numerical result. The ratio between the strong and weak field strengths is set to $\epsilon=$ $\left\{10^{-3}, 10^{-6}\right\}$ (left,right). The vertical red lines are placed at $\tilde{\gamma}=\tilde{\gamma}^{\text {crit }}$. The improved effective reflection point $x_{4}^{\mathrm{p}}=x_{4}^{\mathrm{i}}+\delta / \tilde{\omega}$ has been applied.

ical computation is depicted in Fig. 11. In the previous example, the analytical results are clearly improved. The analytical curve is in good agreement with the exact numerical computation. This observation confirms again the validity of the reflection picture in the case with a poleless weak field.

\section{Super Gaussian}

In the previous examples we found that the standard Gaussian field (7.9) generally leads to more accurate results compared to the sinusoidal field, if we just apply the intersection points as effective reflectors without any further correction $\delta$, cf. App. D. It is expected that a field with a stronger slope in the vicinity of such intersection points will lead to 

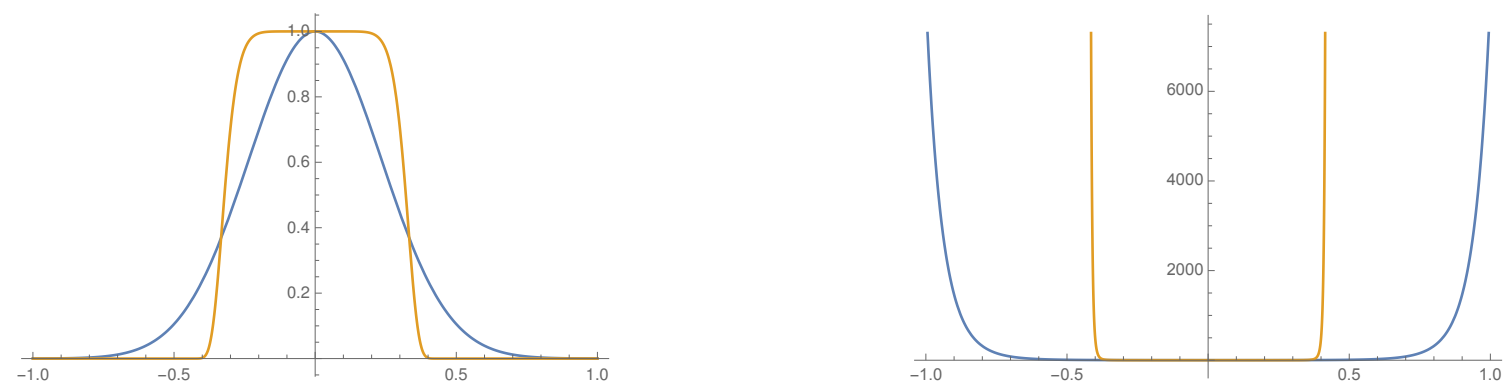

Figure 12. Comparison between the standard (7.9) and super Gaussian (7.29) profiles in blue and yellow, respectively. In the left panel the function $g(t)$ in Lorentzian time is plotted. The right panel shows the analytic continued function $g\left(x_{4}\right)$.

an increase in the accuracy for the analytical prediction. For that reason, let us discuss a third example with a higher order super Gaussian field described by

$$
g(t)=\exp \left(-(\tilde{\omega} t)^{10}\right), \quad G\left(x_{4}\right)=-\frac{\left(\tilde{\omega} x_{4}\right) \mathbf{E}_{\frac{9}{10}}\left(\left(-i \tilde{\omega} x_{4}\right)^{10}\right)}{10} .
$$

The comparison with the previously studied Gaussian field (7.9) is depicted in Fig. 12. The field profile resembles a rectangular potential-wall with a flat top (left panel). Rotating the function $g$ in the complex plane shows a dramatical increase of the curved slope, similar to the situation with a Sauter field. Here, $\mathbf{E}_{n}(z)$ denotes the exponential integral function. The intersection point in the present case is obtained as

$$
x_{4}^{\mathrm{i}}=\frac{(\ln (1 / \epsilon))^{1 / 10}}{\tilde{\omega}} .
$$

Setting $x_{4}^{\mathrm{p}}=x_{4}^{\mathrm{i}}$, leads to the modified invariant

$$
a \approx 4 \frac{\gamma}{\omega} \arcsin \left(\frac{1}{\tilde{\gamma}}(\ln (1 / \epsilon))^{1 / 10}\right)
$$

with the critical Keldysh parameter

$$
\tilde{\gamma}^{\text {crit }}=(\ln (1 / \epsilon))^{1 / 10} \approx(|\ln (\epsilon)|)^{1 / 10} .
$$

Note that the LMA (2.6) condition becomes

$$
m a \approx \frac{E_{\mathrm{S}}}{E} 4 \arcsin \left(\frac{1}{\tilde{\gamma}}(\ln (1 / \epsilon))^{1 / 10}\right) \gg 1 .
$$

For the instanton path we obtain accordingly

$$
\begin{aligned}
& x_{4}(u) \approx \frac{m}{e E} \sin \left(4 u \arcsin \left(\frac{(\ln (1 / \epsilon))^{1 / 10}}{\tilde{\gamma}}\right)\right), \\
& x_{3}(u) \approx \frac{m}{e E} \cos \left(4 u \arcsin \left(\frac{(\ln (1 / \epsilon))^{1 / 10}}{\tilde{\gamma}}\right)\right)-\mathcal{C},
\end{aligned}
$$



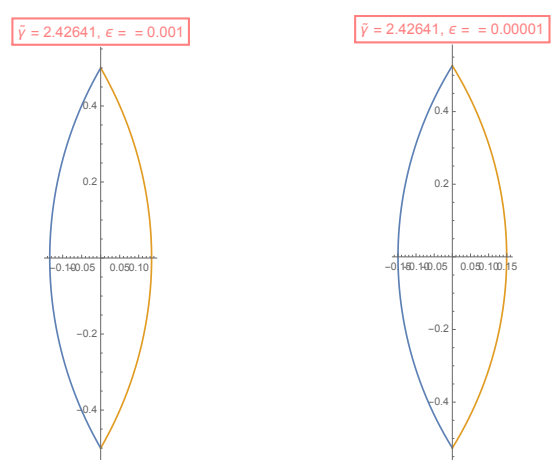

Figure 13. Instanton paths for an electric field as superposition of a strong static and weak super Gaussian field from (7.29) are plotted for $\epsilon=\left\{10^{-3}, 10^{-5}\right\}$ (left,right). The combined Keldysh parameter is set to $\tilde{\gamma}=2 \tilde{\gamma}^{\text {crit }}(\epsilon=0.001)$. The instanton is reflected at the intersection point, i.e. $x_{4}^{\mathrm{p}}=x_{4}^{\mathrm{i}}$.

which is plotted in Fig. 13 for $\epsilon=\left\{10^{-3}, 10^{-6}\right\}$. The constant $\mathcal{C}$ plays the same role as before, i.e. $\mathcal{C}=x_{3}(u= \pm 1 / 4)$. One can observe that the instanton paths do not differ much from each other, i.e. the $\epsilon$ dependence has become weaker. This is in line with the situation for fields which have true poles, like for the Sauter or Lorentzian field, respectively. Because of the strong slope of the curve, the position of the intersection points will be almost fixed and will not change with varying $\epsilon$. Inserting (7.30) in (6.7), one finds accordingly the stationary action

$$
\mathcal{W}_{0} \approx \frac{m^{2}}{e E}\left(\frac{2(\ln (1 / \epsilon))^{1 / 10}}{\tilde{\gamma}^{2}} \sqrt{\tilde{\gamma}^{2}-(\ln (1 / \epsilon))^{1 / 5}}+2 \arcsin \left(\frac{(\ln (1 / \epsilon))^{1 / 10}}{\tilde{\gamma}}\right)\right)
$$

The latter is depicted in Fig. 14. The result agrees well with the numerical curve. Hence,
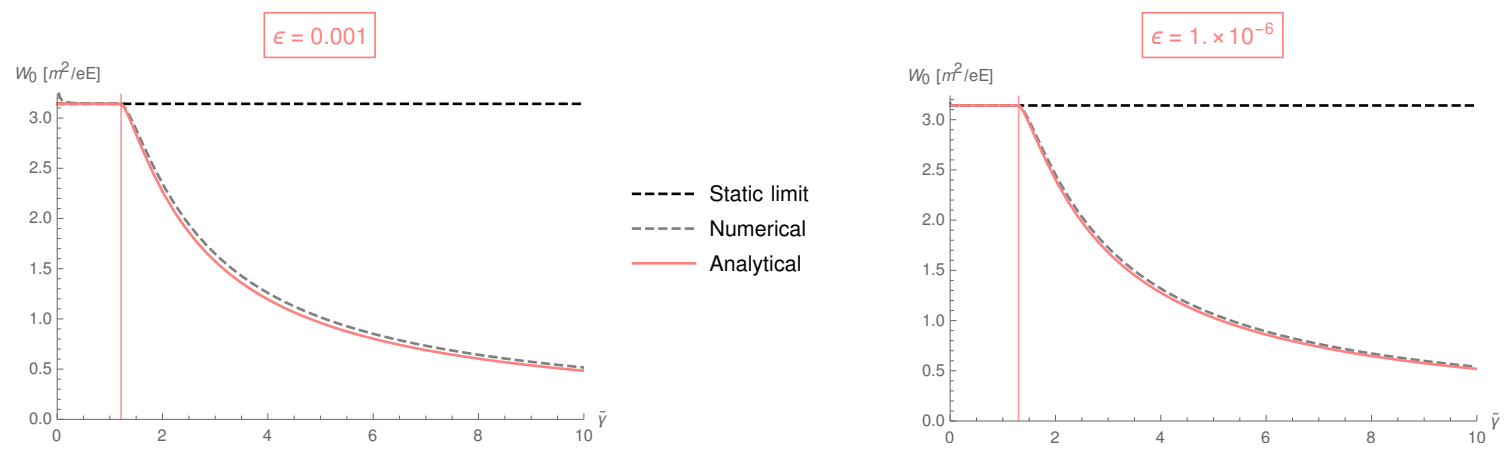

Figure 14. $\mathcal{W}_{0}$ for the weak super Gaussian field (7.29). The analytical prediction (7.35) is compared with the numerical result. Here, the instanton is assumed to be reflected in the intersection point, i.e. $x_{4}^{\mathrm{p}}=x_{4}^{\mathrm{i}}$. The vertical red lines are placed at $\tilde{\gamma}=\tilde{\gamma}^{\text {crit }}$.

the discussed features from above lead indeed to a substantial improvement of the analytical estimation, already with $x_{4}^{\mathrm{p}}=x_{4}^{\mathrm{i}}$. 
The results can be generalised for an arbitrary super Gaussian field described by

$$
g(t)=\exp \left(-(\tilde{\omega} t)^{(4 N+2)}\right), \quad N \in \mathbb{N}_{0} .
$$

The corresponding intersection point is

$$
x_{4}^{\mathrm{i}}=\frac{(\ln (1 / \epsilon))^{1 /(4 N+2)}}{\tilde{\omega}} .
$$

Thus, for $N>2$ we may expect the prediction with $x_{4}^{\mathrm{p}}=x_{4}^{\mathrm{i}}$ to be even more accurate compared to the latter case with $N=2$. Thus, no additional improvements (i.e. $\delta, \Delta$ ) would be needed. Such a higher order super Gaussian field will behave almost as the previously discussed bell-shaped Sauter/Lorentzian fields having poles. The $\epsilon$ dependence will vanish with increasing $N$. The vacuum pair production rate $\mathcal{R}$ will be enhanced even more, simply due to

$$
x_{4}^{\mathrm{p}} \rightarrow 0 \quad(N \rightarrow \infty)
$$

\section{Comparison of $\mathcal{W}_{0}$}

In the following we compare the predicted worldline actions $\mathcal{W}_{0}$ for the fields discussed in the previous sections. We consider both types of weak fields, namely, with and without poles. The results are plotted in Fig. 15 for $\epsilon=\left\{10^{-3}, 10^{-6}\right\}$. The fields without poles
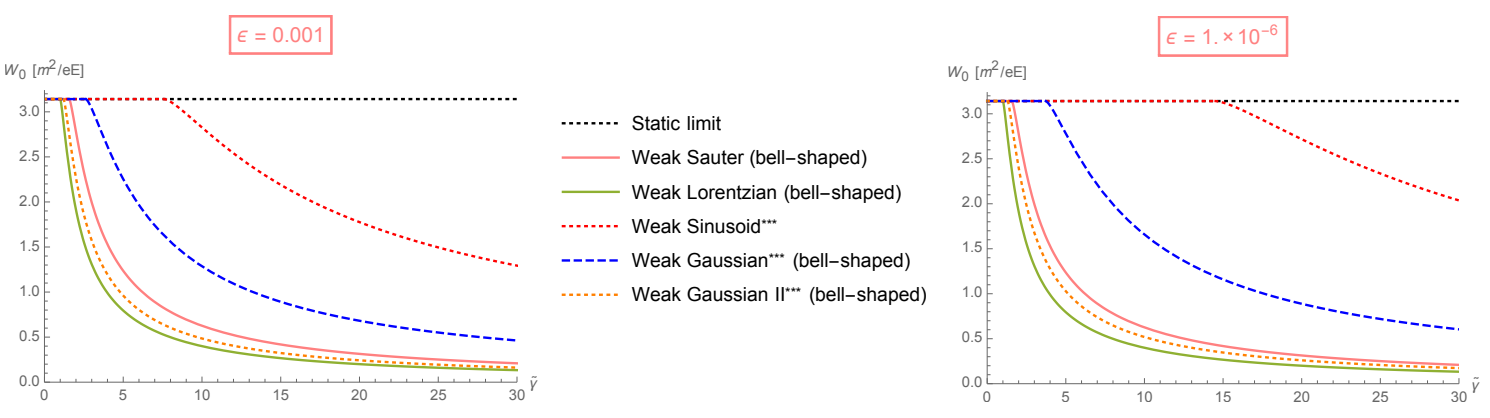

Figure 15. Comparison of the stationary worldline action $\mathcal{W}_{0}$ for different weak field profiles. The ratio between the strong and weak field strengths is set to $\epsilon=\left\{10^{-3}, 10^{-6}\right\}$ (left,right). The profiles are listed in the legend. Fields without poles are marked with asterisks. Note that the applied critical Keldysh parameter for those fields is approximated by $\tilde{\gamma}^{\text {crit }}$. For more on the true critical value we refer to the discussion in the following subsection.

are marked with asterisks in the plot legend. Note that the critical Keldysh parameter for the latter type is determined by the approximate value $\tilde{\gamma}^{\text {crit }}$. More on the true critical parameter will be discussed in the next part. Despite this approximation, we have seen in the discussions before, that with additional improvements for the effective reflection points (Sec. 7) we could indeed obtain good approximate predictions also for fields without poles, cf. Figs. 10 and 11, mainly for the valid regime $\tilde{\gamma}>\tilde{\gamma}^{\text {crit }}$.

Comparing the results in Fig. 15, we observe that bell-shaped fields in general enhance the pair production rate much more than the pure (infinitely extended) oscillatory 
sinusoidal field. Those among them, which have true poles, tend to reduce the stationary action even more. This is shown upon the direct comparison with the Gaussian field, which represents a bell-shaped field, but has no poles. Importantly, the super Gaussian field (7.29) which obviously does not share such a property as well, i.e. has no true poles, leads to comparable enhancement signatures. It is even much more enhancing than the Sauter field which has true poles. The main reason for this counter-intuitive observation is, that this type of field behaves in some sense like the Sauter and Lorentzian fields. This can be understood if we rotate the field in the complex plane. Although, there does not exist any true pole for it, the field function has a very strong slope in the vicinity of the relatively small intersection point (i.e. intersection with the strong static field line), cf. Sec. 7. The latter is almost equal to the (improved) effective reflection point and (true) critical point. Usually, those differ considerably from each other, if the field does not have any distinct pole structure, see below. It is this property of the super Gaussian field, which makes it behaving like the Sauter and Lorentzian field.

The latter discussed bell-shaped fields may have important consequences on oscillatory pulsed fields (wave packets). Such profiles can usually be described by multiplying, for instance, an infinitely extended sinusoidal field with a bell-shaped envelope field, say of Sauter or Gaussian type, respectively. According to the presented results, such a weak field will predominantly trigger the assistance via instanton reflections in the poles of the envelope function. Varying the pulse width via the frequency of the latter is therefore expected to be dominating the enhancement in the dynamically assisted mechanism. However, as we already discussed in Sec. 6, there may be interesting quantum interference effects if we resolve the momentum spectrum of the produced pair, which are generally sensitive to the sub-cycle structure of such a wave packet. This means that the pair production rate is in general highly sensitive to the (ideally) finite size of the weak pulse. This may for sure be substantial for planned laser experiments where fields have usually pulse profiles with very short widths. Taking into account those significant properties, one may think about further improvements to optimise the weak field profile in order to enhance the vacuum pair production rate.

\section{Critical combined Keldysh parameter}

In this section we discuss the critical combined Keldysh parameter in detail. In case of weak fields with a distinct pole structure, we can compute the latter by solving the equation

$$
x_{4}^{\mathrm{p}}=\frac{\gamma}{\omega},
$$

cf. (8.5), which is exact in the relevant regime $\epsilon \ll 1$. However, in situations where true poles are simply not present, we have shown to benefit from the assumption $x_{4}^{\mathrm{p}} \approx x_{4}^{\mathrm{i}}$, deriving the effective critical Keldysh parameter $\tilde{\gamma}^{\text {crit }}$ by calculating the corresponding intersection point. In order to consider $\tilde{\gamma}^{\text {crit }}$ as the critical threshold, we have assumed that the combined electric field after rotation in the complex plane is still described by the horizontal static line, see Figs. 7 and 8, respectively. This is for sure not the realistic situation, since usually there is some contribution from the weak field which leads to an 
increase of the resulting effective field strength due to the superposition of the strong slow field and weak rapid field.

However, it is possible to obtain analytical predictions for the stationary action $\mathcal{W}_{0}$, since the main effect comes from the reflection. One should bear in mind, that further improvements with respect to the effective reflection points were needed. Our analytical predictions for the sinusoidal as well as for the Gaussian weak field coincide well with exact numerical computations, cf. Figs. 10 and 11, at least for $\tilde{\gamma}>\tilde{\gamma}^{\text {crit }}$. These agreements are indeed a strong confirmation of the instanton reflection. In the absence of true poles simple geometrical arguments are generally not very clear. The presented studies have helped therefore to sort previous observations into a more general picture.

However, as we have already discussed, the applied critical value $\tilde{\gamma}^{\text {crit }}$ does not correspond to the true critical threshold value, see Figs. 7 and 8 . This property is a very distinct feature, especially, for fields without true poles. From our previous analysis we expect that the difference between the true critical point and the intersection point will decrease for $\epsilon \rightarrow 0$, see again Figs. 10 and 11. Note that fields with true poles do not lead to such discrepancies. In those cases, intersection, critical and reflection point are just given by the Euclidean pole itself, i.e. $\delta, \Delta \rightarrow 0$, expectably in the regime $\epsilon \ll 1$. This is the reason why poleless weak fields assist less strong for $\tilde{\gamma}$ around the true critical combined Keldysh parameter. In contrast, for fields with true poles the curve for $\mathcal{W}_{0}$ decreases very rapidly as soon as $\tilde{\gamma}$ reaches the true critical value, cf. Fig. 5 .

In Sec. 7 we have briefly discussed what the true critical point is expected to be, cf. (7.16). A detailed discussion on the parameter $\Delta$ and its derivation is presented in App. B. If we take the assumption from (7.16), the difference between the intersection point $x_{4}^{\mathrm{i}}$ and the true critical point becomes

$$
x_{4}^{\mathrm{i}}-(1-\Delta) x_{4}^{\mathrm{i}}=\Delta x_{4}^{\mathrm{i}}=\Delta \frac{\tilde{\gamma}^{\text {crit }}}{\tilde{\gamma}} \frac{m}{e E}
$$

where the value for $\Delta$ has been derived in App. B for the sinusoidal and Gaussian field, cf. (B.6) and (B.9). Those are depicted in Fig. 16 (top-left). The corresponding true critical Keldysh parameter is determined by (7.17). As soon as $\epsilon$ approaches smaller values, $\Delta$ tends to become small as well. This is consistent with our expectation and observations in Figs. 10 and 11.

The resulting true critical combined Keldysh parameter, i.e. $(1-\Delta) \tilde{\gamma}^{\text {crit }}$, is plotted in the bottom panel of Fig. 16. Note that the obtained values coincide very well with the critical behaviour in Figs. 10 and 11. The improved values are of high accuracy, already with computing the correction $\Delta$ up to order $\mathcal{O}\left(\Delta^{2}\right)$, cf. (B.3). A small deviation, however, occurs in case of the sinusoidal field for $\epsilon=10^{-3}$, comparing the analytically predicted value $(1-\Delta) \tilde{\gamma}^{\text {crit }} \approx 4$ marked by the blue dashed curve in Fig. 16 (bottom) with the observed value in Fig. 10 (dashed gray curve, left panel) located around $\tilde{\gamma} \approx 3$. Such a difference originates due to $\Delta \lesssim 1$, cf. top-left panel in Fig. 16. To improve the analytical result, one can truncate the Taylor series in (B.3) after an appropriate higher order in $\Delta$. The very well coincidence in the remaining other cases confirm the validity of (B.6) and (B.9) 


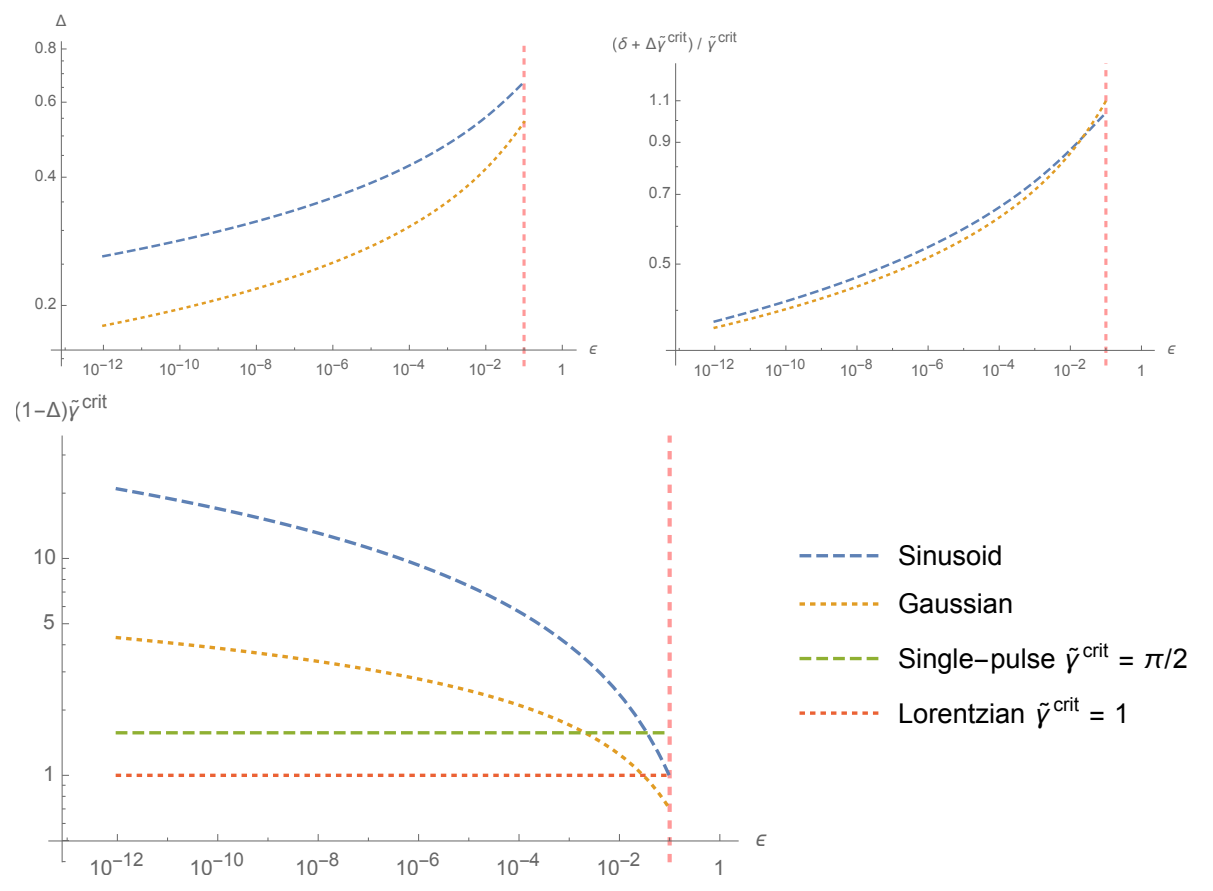

Figure 16. Comparison of $\Delta$ for the sinusoidal and Gaussian field (top-left). The characteristic non-zero difference (with $\tilde{\gamma}=\tilde{\gamma}^{\text {crit }}$ ) between the improved effective reflection point and the true critical point is shown in units of $\frac{m}{e E}$ (top-right). Note that for fields with a distinct pole structure we find $\delta, \Delta \rightarrow 0$ in the relevant regime $\epsilon \ll 1$. The true critical combined Keldysh parameter, i.e. $(1-\Delta) \tilde{\gamma}^{\text {crit }}$, for fields without poles, introduced in (7.17), is plotted in the bottom panel. Note the difference compared to the previous estimations in Fig. 9. The correction $\Delta$ is computed up to the order $\mathcal{O}\left(\Delta^{2}\right)$. For improvements, in particular for the sinusoidal field due to a in general larger $\Delta$, cf. top-left panel, we can simply truncate the Taylor series in (B.3) after higher orders in $\Delta$. The vertical pink dashed line is placed at $\epsilon=0.1$.

as solutions for (A.4) that is in general very challenging to solve analytically because of its non-trivial transcendental structure, see [11, 64] for detailed numerical considerations.

In addition we have plotted the constant lines in Fig. 16 at $\frac{\pi}{2}$ and 1 , which are exact solutions for the Sauter and the Lorentzian field, respectively. For the remaining two poleless fields (sinusoidal and Gaussian) we find the expected non-constant dependence in $\epsilon$. As soon as the latter parameter increases, the true value will be larger compared to the estimation based on $x_{4}^{\mathrm{i}}$. This is also in line with the presented plots in Figs. 10 and 11, respectively. For sufficiently large $\epsilon$, say $\sim 10^{-2}$, we even achieve values below the horizontal constant line for the Sauter field located at $\frac{\pi}{2}$ (green, dashed). However, although in this case the Gaussian field will start to enhance before the bell-shaped Sauter field, it will reduce the stationary action $\mathcal{W}_{0}$ much slower with increasing $\tilde{\gamma}$. This is a direct consequence of the difference between the relatively large (improved) effective reflection point and the true critical point.

Applying the corresponding relations, we obtain for the latter characteristic difference, i.e. improved effective reflection point (7.15) minus true critical point (7.16), in units of 
$\frac{m}{e E}$,

$$
\frac{\delta+\Delta \tilde{\gamma}^{\text {crit }}}{\tilde{\gamma}}
$$

Using (7.20) and (B.6) (sinusoidal field), (7.25) and (B.9) (Gaussian field), we have plotted the corresponding curves for $\tilde{\gamma}=\tilde{\gamma}^{\text {crit }}$ in Fig. 16 (top-right). On the contrary, note that already for $\epsilon \sim 10^{-2}$ the last expression in (7.41) vanishes completely if the considered field has a true pole structure, like in the case of a Sauter or Lorentzian field, respectively.

From those observations we conclude that in the relevant regime $\epsilon \ll 1$ the critical combined Keldysh parameter for fields with true poles determines exactly the point where the weak field contribution becomes essential and where the reflection sets in, both effects apply exactly at the same point. However, if poles are not present, the true critical point does not, in general, correspond to the effective reflection point which usually has a much larger value. Thus, in the latter case the reduction of $\mathcal{W}_{0}$ progresses much slower for $\tilde{\gamma}$ below $\tilde{\gamma}^{\text {crit }}$ that determines the point where both fields contribute equally as we can clearly see for instance in Fig. 10. The reason is that the enhancement is mainly triggered by the increased effective field strength due to the superposition of the strong and weak field, see comparison between Lorentzian field (left) and sinusoidal field (right) cases in Fig. 7. This is somewhat similar to the situation with a single-mode inhomogeneous electric field, see Fig. 19 (only gray solid line) in App. C. The large valued effective reflection point is too far away from the true critical point. However, as soon as both points merge together, i.e. $(7.41) \rightarrow 0$, which happens for sufficiently large $\tilde{\gamma}>\tilde{\gamma}^{\text {crit }}$, the enhancement becomes stronger due to a smaller effective reflection point. Thus, the decrease of $\mathcal{W}_{0}$ evolves more quickly, similar to the case with true poles acting as reflectors with relatively small values. Basically, this effect turns out to be the major attribute that distinguishes these two types of fields.

\section{Critical inhomogeneity}

In Sec. 5 we could directly read off one special critical point from the external part of the worldline action which we denoted as $x_{4}^{*}$. It was determined by, cf. (5.9),

$$
x_{4}^{*}=F^{-1}\left(\frac{\gamma}{\omega}\right)
$$

which corresponds to $\dot{x}_{3}=0$. Closing the instanton in $x_{4}^{*}$ results in the usual decay process which is only driven by the strong field. For the dynamically assisted mechanism, however, we need the instanton to be closed at the pole of the weak fast field. Then, the instanton will be squeezed due to its reflection. Therefore, one cannot demand a vanishing velocity in the closing point of the instanton path, i.e. $\dot{x}_{4} \neq 0$. Therefore, the critical point in (8.1) cannot be permitted as closing point for the dynamically assisted mechanism. This requirement is exactly what is demanded with the reflection condition (5.10). However, from the equality

$$
x_{4}^{*}=x_{4}^{\mathrm{p}},
$$


we can obtain immediately the critical frequency

$$
\tilde{\omega}^{\text {crit }}=\frac{e E}{m} \tilde{\gamma}^{\text {crit }}
$$

for the weak field. Below this value the latter will not lead to an enormous enhancement via dynamical assistance. As we have seen in case a weak Sauter field is superimposed with a strong static field, the critical value is given by

$$
\tilde{\omega}^{\text {crit }}=\frac{\pi m}{2} \frac{E}{E_{\mathrm{S}}}, \quad \tilde{\gamma}^{\text {crit }}=\frac{\pi}{2},
$$

cf. (6.11). For the weak Lorentzian field this critical value is $\tilde{\gamma}^{\text {crit }}=1$, cf. (6.16). On the other hand, (8.2) clearly shows that the critical inhomogeneity $\tilde{\gamma}^{\text {crit }}$ or frequency $\tilde{\omega}^{\text {crit }}$, respectively, has to depend on the strong field, which determines the LHS of the condition (8.2), and on the weak field itself, which is responsible for the RHS of the condition. This is expected, since with increasing strong field inhomogeneity $\gamma$ the closing point $x_{4}^{\mathrm{c}}$

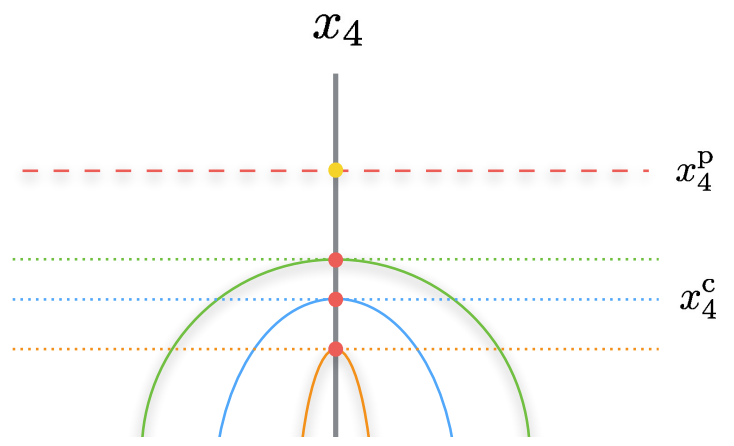

Figure 17. Condition for the assisted mechansim: in case of reflection both critical points, i.e. closing point of the instanton (red dots) and the weak field pole (yellow dot), have to coincide, cf. (8.2). For increasing strong field inhomogeneity the closing points drift towards the origin (red dots) and the instanton (solid lines) curves much stronger around its closing point.

will drift towards the origin along the complex time axis. This situation is sketched in Fig. 17. During this shift one should note that the curvature of the instanton will become larger around the closing point $x_{4}^{\mathrm{c}}$. According to this picture, the pole $x_{4}^{\mathrm{p}}$ of the weak field becomes smaller for being located in the same point as $x_{4}^{\mathrm{c}}$. This is mandatory for the instanton reflection, cf. condition (5.10). Therefore, the critical combined Keldysh parameter $\tilde{\gamma}^{\text {crit }}$ depends on $\gamma$ and on the strong field profile. Such a dependence for the weak field inhomogeneity has been analytically obtained for the case of a strong spatial Sauter field combined with a temporal Sauter field, treating both dependencies nonperturbatively [77]. Following analogous geometric arguments as we have discussed here, it will be promising to extend these analytical studies also to spatiotemporal electric backgrounds $\boldsymbol{E}(t, x)$ with sinusoidal or (super) Gaussian temporal dependence. Such considerations will be presented in forthcoming studies [91]. 
For too large $\gamma$ the strong field will drive the vacuum decay alone, since the threshold $\tilde{\gamma}^{\text {crit }}$ will be comparable large. This corresponds to the usual anti-adiabatic perturbative multi-photon process. In the intermediate regime, i.e. not too large field inhomogeneity $\gamma$, one expects a window where the weak field with moderate $\tilde{\gamma}$ triggers the assistance. However, as soon as $\gamma$ becomes much smaller than unity, the (locally) static strong field will be again a good approximation, i.e its profile becomes again irrelevant. Nevertheless, an explicit example for which the strong field is assumed in the anti-adiabatic multi-photon regime, i.e. non-static, is studied in App. C for illustrative reasons and for confirming the validity of the reflection picture.

In the described situation above, the general question may be, whether the present reflection picture is valid or not if one allows for $\gamma$ values of order unity or larger. Note, however, that this situation is in general not relevant and realistic for upcoming experimental designs.

To resolve the latter question, let us bear in mind that the basic starting point for the reflection picture was based on the negligibility of the weak field contribution away from the reflection point in the original instanton equations (4.4). Therefore, even if we allow $\gamma$ to be large, there will be always a reflector from the much more rapid weak field, which dominates above the critical threshold $\tilde{\gamma}^{\text {crit }}$. Also for $\epsilon \ll 1$, those poles will be much closer to the origin than, if present, the strong field poles, because of the assumed hierarchy of frequencies $\tilde{\omega} \gg \omega$.

In order to study the strong field profile dependence of the critical threshold, we use the relation in (8.1), and the criticality condition is given by

$$
\gamma=\omega F\left(x_{4}^{\mathrm{p}}\right) .
$$

For illustrative reasons, let us assume the weak field to be of Lorentzian type for which we introduced previously the relevant functions (6.13), i.e.

$$
g(t)=\frac{1}{\left(1+(\tilde{\omega} t)^{2}\right)^{3 / 2}}, \quad G\left(x_{4}\right)=\frac{x_{4}}{\sqrt{1-\left(\tilde{\omega} x_{4}\right)^{2}}},
$$

with the pole (6.14) being located in $x_{4}^{\mathrm{p}}=\frac{1}{\tilde{\omega}}$. Using this setup we can compute for several strong field profiles the critical combined Keldysh parameter $\tilde{\gamma}^{\text {crit }}$, starting from (8.5) using the relation $\omega=\frac{m \gamma E}{E_{\mathrm{S}}}$ :

Static field $\quad f(t)=1, \quad F\left(x_{4}\right)=x_{4}$,

$$
\tilde{\omega}^{\text {crit }}=m \frac{E}{E_{\mathrm{S}}}, \quad \tilde{\gamma}^{\text {crit }}=1
$$

Lorentzian field $\begin{array}{rlrl}f(t) & =\frac{1}{\left(1+(\omega t)^{2}\right)^{3 / 2}}, & & F\left(x_{4}\right)=\frac{x_{4}}{\sqrt{1-\left(\omega x_{4}\right)^{2}}}, \\ \tilde{\omega}^{\text {crit }} & =m \frac{E}{E_{\mathrm{S}}} \sqrt{1+\gamma^{2}}, & \tilde{\gamma}^{\text {crit }}=\sqrt{1+\gamma^{2}}\end{array}$ 


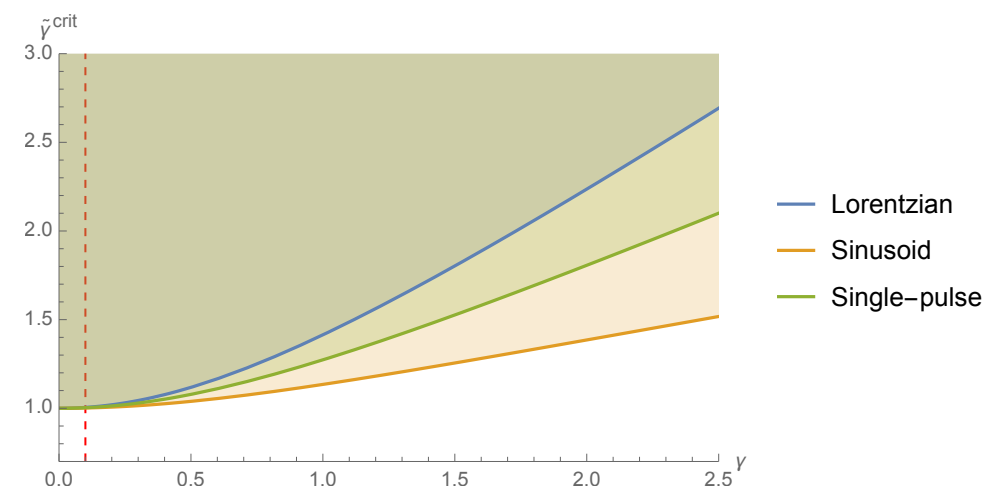

Figure 18. Critical combined Keldysh parameter $\tilde{\gamma}^{\text {crit }}$ for the case of a weak Lorentzian field superimposed with a strong field of different profiles. The profiles are listed in the legend. The relevant regime for the dynamically assisted mechanism $\left(\tilde{\gamma}>\tilde{\gamma}^{\text {crit }}\right)$ is depicted by the colored region. The adiabatic nonperturbative regime, i.e. $\gamma \ll 1$, lies to the left of the vertical dashed red line where $\tilde{\gamma}^{\text {crit }} \approx 1$.

Sinusoidal field $\quad \begin{aligned} f(t) & =\cos (\omega t), \quad F\left(x_{4}\right)=\frac{\sinh \left(\omega x_{4}\right)}{\omega}, \\ \tilde{\omega}^{\text {crit }} & =m \frac{E}{E_{\mathrm{S}}} \frac{\gamma}{\operatorname{arcsinh}(\gamma)}, \quad \tilde{\gamma}^{\text {crit }}=\frac{\gamma}{\operatorname{arcsinh}(\gamma)},\end{aligned}$

Sauter field $\quad f(t)=\operatorname{sech}^{2}(\omega t), \quad F\left(x_{4}\right)=\frac{\tan \left(\omega x_{4}\right)}{\omega}$,

$$
\tilde{\omega}^{\text {crit }}=m \frac{E}{E_{\mathrm{S}}} \frac{\gamma}{\arctan (\gamma)}, \quad \tilde{\gamma}^{\text {crit }}=\frac{\gamma}{\arctan (\gamma)} .
$$

For $\gamma \rightarrow 0$ we approach for all cases the static limit (8.8) which is the adiabatic nonperturbative tunneling regime. However, for $\gamma$ becoming larger, the critical value $\tilde{\gamma}^{\text {crit }}$ for the combined Keldysh parameter increases first parabolic then linear in $\gamma$, cf. Fig. 18. Note that the relevant regime for the dynamically assisted mechanism, i.e. where $\tilde{\gamma}>\tilde{\gamma}^{\text {crit }}$, is indicated by the colored patterns above the curves. For small values $\gamma>0.1$, region right to the vertical dashed red line, we leave the region with almost constant dependence in $\gamma$ for the critical Keldysh parameter, i.e. $\tilde{\gamma}^{\text {crit }} \approx 1$. The latter provides the approximate nonperturbative regime for the strong field. The slope of the plotted curves turn out to be much stronger for profiles that lead in general to smaller reflection points: in case of such field profiles, the inhomogeneity of the weak field has to be much larger, for reflecting the instanton and assisting the vacuum decay. Otherwise the enhancement will be only determined by the strong field inhomogeneity.

Those studies exhibit in principal the two types of mechanisms which lead to a substantial enhancement of the vacuum decay rate in temporally inhomogeneous electric fields:

1. The enhancement is driven by a single temporally inhomogeneous electric field (anti-adiabatic perturbative multi-photon regime). The role of a second weak field becomes negligible with increasing $\gamma$. A characteristic threshold in this case does not exist. 
2. The background is composed of a strong slow field (adiabatic nonperturbative tunneling regime) superimposed with a weak rapid field. This situation corresponds to the dynamically assisted Schwinger mechanism. The contribution of the weak rapid field is essential for the enhancement. It sets in for $\tilde{\gamma}$ above the characteristic critical Keldysh parameter $\tilde{\gamma}^{\text {crit }}$.

\section{Summary}

In this paper we have discussed vacuum pair production in temporally inhomogeneous electric backgrounds composed of a strong slowly varying field and a weak field altering at a much shorter time scale. Such type of combined backgrounds can trigger an enormous enhancement of the vacuum pair production rate, known as the so-called dynamically assisted Schwinger mechanism. The purpose in the present paper is to work out main features of this mechanism.

Using the worldline formalism, we have obtained from the stationary instanton equations two separate critical points. While one of them is responsible for the closing of the instanton path, the other serves as a reflecting mirror in Euclidean spacetime. Employing this reflection picture, we have analysed characteristic features of this dynamical mechanism considering various types of electric backgrounds. Specifically, we have focused on the role of the assisting weak rapid field and the influence of its profile. Based on geometric considerations, we explain the origin for substantial differences. For this, we have distinguished between two types of backgrounds.

The first type of weak fields are those which could be characterised by a distinct pole structure after rotation in the complex plane. This is the case where geometrical arguments are very intuitive. Consistent with previous observations, we have shown that in the weak limit, i.e. $\epsilon \ll 1$, the drastic enhancement turns out to be the direct consequence of instanton reflections in such poles. Beginning with the well-studied Sauter field, we have also considered the case with a weak rapid field of Lorentzian type. We have shown that these behave similarly. The reason for this common feature is caused due to their similar pole structure and not due to their almost indistinguishable bell-shaped profiles. In this context, we have also discussed the impact of a possible sub-cycle structure of the weak rapid field. Performing explicit computations in this reflection picture, we have illustrated that the assistance is primarily determined by the pole of the bell-shaped envelope function which is chosen to be Lorentzian. Only with sufficiently large $\epsilon$ the encased sub-cycle structure of the considered oscillatory pulse field leads to considerable deviations.

The second type is characterised by fields without a distinct pole structure. Based on analogous geometrical arguments, we have obtained specific conditions from combined analysis based on the instanton equations and the equivalent WKB approach. By doing so, we have been able to compute analytically the corresponding effective reflection points. In addition, we have also computed analytically the true critical threshold given by the combined Keldysh parameter for such poleless fields. In contrast to the latter type, we have shown that the true critical point ${ }^{10}$, which determines the mentioned characteristic

\footnotetext{
${ }^{10}$ This is the point where the weak field starts to contribute.
} 
threshold, deviates from the relatively large valued effective reflection point, even in the highly weak limit. This feature turns out to be the major difference between fields with and without poles. In the former case the reflection and the critical point turn out to be equal to the pole itself. We have demonstrated that this discrepancy can be seen as the primary reason why poleless fields, as for instance the sinusoidal field, enhance the vacuum decay less than fields with poles.

Furthermore, we have shown that the additional $\epsilon$ dependence in this mechanism occurs if the weak rapid field can not be characterised by a distinct pole structure. However, for poleless fields with a steeply rising rotated profile, as, for instance, the discussed super Gaussian type, such a dependence becomes increasingly suppressed. These insights have clearly shown that the influence of the weak rapid field in assisted vacuum pair production substantially differs for field profiles with and without a distinct pole structure.

We have also confirmed the validity of the reflection picture by explicit analytical calculations even when the strong field inhomogeneity is assumed to be non-negligible compared to the faster weak field. The predictions in the relevant regime are in perfect agreement with numerical computations.

Conclusively, we have seen that the dynamical assistance is predominantly determined by instanton reflections, no matter whether poles are present or not. The presented studies have confirmed the reflection picture also in the latter less intuitive case. For the presented examples, we have demonstrated that the exact shape of the field profile does not serve as the main regulator, at least in the highly weak limit. The location of characteristic critical points determines the strength of the assistance triggered by the weak rapid field. We may conclude that reflection points close to the origin lead basically to stronger enhancement signatures. Such insights may allow to pursue further optimisation studies with respect to the weak field in order to maximize these effects.

One should note that we have not addressed quantum interference effects in the presented studies. Those become manifest in the momentum spectrum of the produced pairs. In order to demonstrate those signatures, one needs to incorporate additional information of the phase-space.

It is also interesting to work out analog geometric considerations for the case of an additional spatially inhomogeneous field. This will allow an analytical treatment for electric backgrounds with spatiotemporal dependence. Furthermore, it may facilitate in particular the role of such backgrounds with regard to the nonlocal nature of vacuum pair production. Such considerations will be the subject of forthcoming studies [91].

\section{Acknowledgments}

We are grateful to Holger Gies for valuable comments and a careful reading of the manuscript. We thank Andreas Ringwald for support and valuable comments during this work. IA thanks Dennis D. Dietrich for interesting discussions. The authors acknowledge the support of the Colloborative Research Center SFB 676 Particles, Strings and the Early Universe of the DFG. 


\section{A Improved mirrors: $\delta$ correction}

Weak fields without a distinct pole structure have generally a finite curve slope in the vicinity of the intersection points. In contrast, fields with true poles form almost vertical reflectors which intersect with the strong field curve. Thus, their position is almost fixed, i.e. it does not alter with the field strength parameter $\epsilon$. Therefore, for the former type of fields, it is required to increase the accuracy for the estimated position of the effective reflection points, because the rate is highly sensitive to those.

Beginning with basic steps in the equivalent WKB approximation, the plan is to obtain conditions that we can combine with our previous analysis in the (worldline) instanton approach to improve our analytical estimations. Moreover, this will illustrate the equivalence between both methods with respect to the (semiclassical) tunneling exponential. For this, both methods lead in general to the same result if the momentum spectrum is peaked around zero (canonical) momentum [66], as it is the case for fields with one spacetime coordinate as considered in this paper, see e.g. [84].

Starting with the Dirac equation in a classical electromagnetic field, one can first identify the evolution of the corresponding Bogoliubov coefficients. Afterwards the resulting system, which is described by the Riccati equation, can approximately be integrated and one obtains for $p=0$ the condition

$$
i e\left(E F\left(x_{4}^{*}\right)+\tilde{E} G\left(x_{4}^{*}\right)\right)=i m
$$

for the singularities $x_{4}^{*}$, e.g. cf. [11, 84]. This relation can alternatively be derived from the instanton equations (4.4). The singularities $x_{4}^{*}$ determine the pair production probability, at least the correct exponent ${ }^{11}$. The solution of this very often transcendental ${ }^{12}$ equation gives then the poles of the fields. If $\epsilon \ll 1$ and $G$ is sufficiently small, one gets the usual strong field pole $x_{4}^{*}=\frac{m}{e E}$ if $F\left(x_{4}\right)=x_{4}$. For the dynamically assisted mechanism we demand the weak field pole to be dominant. Hence, we have to consider the situation, where the smallness of $\epsilon$ is counterbalanced by the reflection point. This happens in case of setting

$$
\epsilon G\left(x_{4}^{\mathrm{p}}\right) \stackrel{!}{=} \frac{\tilde{\gamma}}{\tilde{\omega}}
$$

where $F$ is assumed to be negligible small in the reflection point, i.e.

$$
F\left(x_{4}^{\mathrm{p}}\right) \ll \epsilon G\left(x_{4}^{\mathrm{p}}\right) .
$$

Note that from the important condition

$$
F\left(x_{4}\right) \stackrel{!}{=} \epsilon G\left(x_{4}\right),
$$

one determines the point in which both fields contribute equally. One should note that equation (A.4) can be of transcendental type (e.g. for Gaussian fields, cf. Sec. 7) which

\footnotetext{
${ }^{11}$ The quantum fluctuation prefactor cannot be correctly determined via WKB, except the (semiclassical) exponential dependence.

${ }^{12}$ In this case a closed-form solution does not exist.
} 
cannot be solved algebraically. In this case, we follow an approximate approach leading to a drastic simplification of the problem, discussed in detail below and in App. B. Note that deriving the latter equation after $x_{4}$ on both hand sides, leads to the intersection condition we have already introduced in (7.2). If the inverse of $G$ does exist, the equation (A.2) can be solved directly leading to the solution

$$
x_{4}^{\mathrm{p}}=G^{-1}\left(\frac{\tilde{\gamma}}{\epsilon \tilde{\omega}}\right) .
$$

This gives us the improved effective reflection point. Reminding that we have started from the assumption $x_{4}^{\mathrm{p}} \approx x_{4}^{\mathrm{i}}$, we try to improve the reflection point via the ansatz

$$
x_{4}^{\mathrm{p}}=x_{4}^{\mathrm{i}}+\frac{\delta}{\tilde{\omega}} .
$$

The constant $\delta>0$ is then determined by

$$
\delta=\tilde{\omega} G^{-1}\left(\frac{\tilde{\gamma}}{\epsilon \tilde{\omega}}\right)-\tilde{\omega} x_{4}^{\mathrm{i}}
$$

It is important to note that, according to the present approach, the intersection point $x_{4}^{\mathrm{i}}$ still has to determine the critical value for the combined Keldysh parameter $\tilde{\gamma}^{\text {crit }}$ (the true value is for sure different from this estimation, cf. App. B), i.e.

$$
\gamma \stackrel{!}{=} \omega F\left(x_{4}^{\mathrm{i}}\right) \hookrightarrow \tilde{\gamma}^{\mathrm{crit}}
$$

Contributions from the weak field have been neglected before the instanton reflection, since the drastic enhancement is caused by such reflections. However, the improved effective reflection point $x_{4}^{\mathrm{p}}$ (A.6) will for sure alter the critical Keldysh parameter from above. This value we denote as $\tilde{\gamma}^{\mathrm{p} \text {,crit }}$ which is determined by the condition

$$
\gamma \stackrel{!}{=} \omega F\left(x_{4}^{\mathrm{p}}\right) \hookrightarrow \tilde{\gamma}^{\mathrm{p}, \mathrm{crit}} .
$$

In order to keep the critical Keldysh parameter as the one determined by the first condition (A.8), one has to shift $\tilde{\gamma}$ in the final expressions via the previous displacement parameter $\delta$,

$$
\tilde{\gamma} \rightarrow \tilde{\gamma}+\delta
$$

The parameter $\delta$ from (A.7) becomes now the difference between the modified and the original critical Keldysh parameter, i.e.

$$
\delta=\tilde{\gamma}^{\mathrm{p}, \text { crit }}-\tilde{\gamma}^{\mathrm{crit}} .
$$

Because of $\delta>0$ and $\epsilon \ll 1$, one can assume

$$
2 \tilde{\gamma}^{\text {crit }}>\tilde{\gamma}^{\text {p,crit }}>\tilde{\gamma}^{\text {crit }}
$$


Note that the last two steps are justified only if the weak field function raises sufficiently fast in the vicinity of the intersection points. Compared to the exact result (A.7), we will therefore neglect the explicit $\tilde{\gamma}$ dependence and rewrite $\tilde{\gamma}^{\text {,crit }}$ as

$$
\tilde{\gamma}^{\mathrm{p}, \text { crit }}=(1+\xi) \tilde{\gamma}^{\mathrm{crit}}
$$

with $0<\xi<1$. Using this approximation, we get for the displacement parameter

$$
\delta=\xi \tilde{\gamma}^{\mathrm{crit}}
$$

still requiring the value for the small parameter $\xi$. Combining (A.6) and (A.7), we get from expression (A.9)

$$
\tilde{\gamma}^{\mathrm{p}, \mathrm{crit}}=\tilde{\omega} G^{-1}\left(\frac{\tilde{\gamma}^{\mathrm{p}, \mathrm{crit}}}{\epsilon \tilde{\omega}}\right)
$$

where $F\left(x_{4}\right)=x_{4}$ has been assumed for the strong slow field $(\gamma \rightarrow 0)$. Subsequently, we use (A.13) to obtain the equation

$$
(\xi+1) \tilde{\gamma}^{\text {crit }}=\tilde{\omega} G^{-1}\left(\frac{(\xi+1) \tilde{\gamma}^{\text {crit }}}{\epsilon \tilde{\omega}}\right) .
$$

Note that the original critical Keldysh parameter $\tilde{\gamma}^{\text {crit }}$ is known from previous analysis based on the intersection points, cf. condition (A.8). The latter equation is difficult to solve in general, because of the nonlinear $\xi$ dependence on the RHS. However, since we have $\xi<1$, one may Taylor expand the nonlinearity in the lowest relevant order and compute $\xi$. This solution can be used to obtain the displacement parameter from the expression (A.14). This is a powerful way to compute $\delta$, specifically, in situations where the inverse function of $G$ is difficult to find or does not exist at all, respectively. Finally, all relevant modifications we need to apply for improving the analytical predictions with respect to the effective reflectors are

$$
\begin{aligned}
x_{4}^{\mathrm{p}} & \rightarrow x_{4}^{\mathrm{i}}+\frac{\delta}{\tilde{\omega}}, \\
\tilde{\gamma} & \rightarrow \tilde{\gamma}+\delta .
\end{aligned}
$$

\section{B Critical Keldysh parameter: $\Delta$ correction}

In App. A we have computed the correction for the effective reflection point. For the case, where the inverse function $G^{-1}$ is complicated to handle, we applied the exact intersection point $x_{4}^{\mathrm{i}}$ determined by (7.2) to obtain the approximate displacement parameter $\delta$. In this section we derive the correction $\Delta$ in (7.16), following an equivalent ansatz based on an appropriate perturbation around the intersection point $x_{4}^{\mathrm{i}}$. The parameter $\Delta$ determines the true critical point (in contrast to the intersection point which determined the criticality so far) where the weak field starts to contribute considerably. The relevant equation to solve is (A.4). We rewrite the true critical point as

$$
(1-\Delta) x_{4}^{\mathrm{i}}
$$


with $0<\Delta<1$, being reasonable for the present purpose, cf. e.g. Figs. 7 and 8 . With this, the original equation becomes

$$
(1-\Delta) x_{4}^{\mathrm{i}}=\epsilon G\left((1-\Delta) x_{4}^{\mathrm{i}}\right)
$$

where we have assumed $F\left(x_{4}\right)=x_{4}$. In contrast to the initial equation above, the latter can be Taylor expanded on the RHS, since $\Delta<0$. Hence, we find the series

$$
(1-\Delta) x_{4}^{\mathrm{i}}=G\left(x_{4}^{\mathrm{i}}\right)-G^{\prime}\left(x_{4}^{\mathrm{i}}\right) \Delta-\frac{1}{2} G^{\prime \prime}\left(x_{4}^{\mathrm{i}}\right) \Delta^{2}+\mathcal{O}\left(\Delta^{3}\right) .
$$

This equation can be solved approximately by truncating the series after a sufficient order in $\Delta$, in the following applied for the sinusoidal and Gaussian field.

\section{Weak sinusoidal field}

The sinusoidal field is known from (7.3). Plugging into (B.2) leads to

$$
(1-\Delta) x_{4}^{\mathrm{i}}=\frac{\epsilon}{\tilde{\omega}} \sinh \left((1-\Delta) \tilde{\omega} x_{4}^{\mathrm{i}}\right) .
$$

With the intersection point (7.4), we obtain up to order $\mathcal{O}\left(\Delta^{2}\right)$,

$$
\tilde{\gamma}^{\text {crit }}(1-\Delta) \approx \epsilon\left(\frac{1}{2} \tilde{\gamma}^{\text {crit }} \Delta^{2} \sinh \left(\tilde{\gamma}^{\text {crit }}\right)-\tilde{\gamma}^{\text {crit }} \Delta \cosh \left(\tilde{\gamma}^{\text {crit }}\right)+\sinh \left(\tilde{\gamma}^{\text {crit }}\right)\right)
$$

Here, we have used the relation $\tilde{\omega} x_{4}^{\mathrm{i}}=\tilde{\gamma}^{\text {crit }}$. The parameter $\Delta$ is then determined by

$$
\begin{aligned}
\Delta & \approx-\frac{\operatorname{csch}\left(\tilde{\gamma}^{\text {crit }}\right)}{\tilde{\gamma}^{\text {crit }} \epsilon}+\frac{\operatorname{coth}\left(\tilde{\gamma}^{\text {crit }}\right)}{\tilde{\gamma}^{\text {crit }}} \\
& +\frac{\operatorname{csch}\left(\tilde{\gamma}^{\text {crit }}\right) \sqrt{\left(2 \tilde{\gamma}^{\text {crit }}-2 \tilde{\gamma}^{\text {crit }} \epsilon \cosh \left(\tilde{\gamma}^{\text {crit }}\right)\right)^{2}-4 \tilde{\gamma}^{\text {crit }}{ }^{2} \epsilon \sinh \left(\tilde{\gamma}^{\text {crit }}\right)\left(2 \epsilon \sinh \left(\tilde{\gamma}^{\text {crit }}\right)-2 \tilde{\gamma}^{\text {crit }}\right)}}{2 \tilde{\gamma}^{\text {crit }^{2}} \epsilon} .
\end{aligned}
$$

\section{Weak Gaussian field}

The field has been already introduced in (7.9). The intersection point is given in (7.10). As before, we plug the corresponding quantities into (B.2) and obtain

$$
(1-\Delta) \tilde{\omega} x_{4}^{\mathrm{i}}=\epsilon \frac{\sqrt{\pi}}{2} \operatorname{erfi}\left((1-\Delta) \tilde{\omega} x_{4}^{\mathrm{i}}\right) .
$$

This equation can be written as (up to order $\left.\mathcal{O}\left(\Delta^{2}\right)\right)$

$$
(1-\Delta) \tilde{\gamma}^{\text {crit }} \approx \epsilon\left(-\frac{2\left(e^{\tilde{\gamma}^{\text {crit }}{ }^{2}} \tilde{\gamma}^{\text {crit }}\right) \Delta}{\sqrt{\pi}}+\frac{2 e^{\tilde{\gamma}^{\text {crit }}{ }^{2}} \tilde{\gamma}^{\text {crit }^{3}} \Delta^{2}}{\sqrt{\pi}}+\operatorname{erfi}\left(\tilde{\gamma}^{\text {crit }}\right)\right)
$$

and we derive

$$
\begin{aligned}
\Delta & \approx \frac{e^{-\tilde{\gamma}^{\text {crit }^{2}}} \sqrt{\left(\sqrt{\pi} \tilde{\gamma}^{\text {crit }}-2 e^{\text {crit }^{2}} \tilde{\gamma}^{\text {crit }} \epsilon\right)^{2}-8 e^{\text {crit }^{2}} \tilde{\gamma}^{\text {crit }^{3}} \epsilon\left(\sqrt{\pi} \epsilon \operatorname{erfi}\left(\tilde{\gamma}^{\text {crit }}\right)-\sqrt{\pi} \tilde{\gamma}^{\text {crit }}\right)}}{4 \tilde{\gamma}^{\text {rit }^{3} \epsilon}} \\
& -\frac{\sqrt{\pi} e^{-\tilde{\gamma}^{\text {crit }^{2}}}}{4 \tilde{\gamma}^{\text {crit }^{2}} \epsilon}+\frac{1}{2 \tilde{\gamma}^{\text {crit }^{2}}} .
\end{aligned}
$$




\section{Non-static strong field}

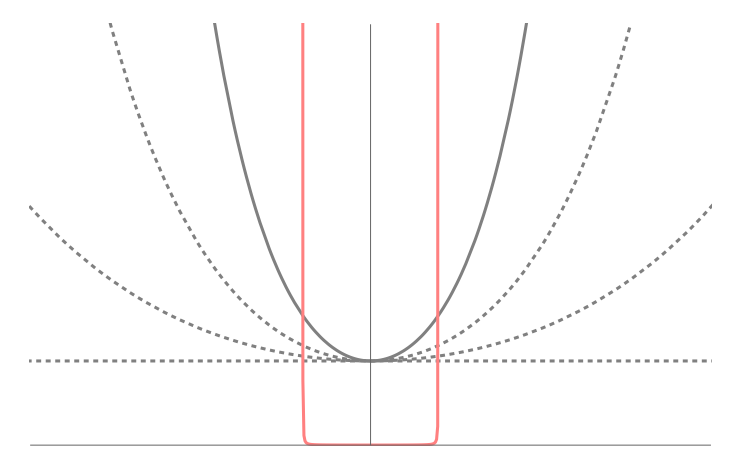

Figure 19. Combined electric background after rotation in the complex plane: the strong inhomogeneous field (without poles, grey solid curve) and weak inhomogeneous field (with poles, pink curve) for $\gamma / \tilde{\gamma}<1$ are plotted separately. Dotted gray curves indicate the increase of $\gamma$ starting at $\gamma=0$ (horizontal dotted line).

Let us illustrate the effects from Sec. 8 with an example. The strong field we assume to be of sinusoidal type and the weak field with Lorentzian profile, i.e.

$$
\begin{aligned}
& f(t)=\cos (\omega t), \quad F\left(x_{4}\right)=\frac{\sinh \left(\omega x_{4}\right)}{\omega}, \\
& g(t)=\frac{1}{\left(1+(\omega t)^{2}\right)^{3 / 2}}, \quad G\left(x_{4}\right)=\frac{x_{4}}{\sqrt{1-\left(\omega x_{4}\right)^{2}}} .
\end{aligned}
$$

The corresponding modification in comparison to the previously studied cases with a static strong field is depicted schematically in Fig. 19. With increasing $\gamma$ we left the static limit by bending up the initial horizontal line (dotted gray curves), representing the function $f\left(x_{4}\right)$. For $\gamma \neq 0$ there will be a substantial structure (solid gray curve) between the poles of the Lorentzian field (pink curve). The interplay between this parabolic strong field curve and the reflecting weak field poles are computed in the present reflection picture. The pole for the weak Lorentzian field is $x_{4}^{\mathrm{p}}=1 / \tilde{\omega}$. Using the expression in (5.14) we get

$$
a=-i \frac{4}{\omega} \mathbf{F}\left(i \frac{\gamma}{\tilde{\gamma}} \mid \frac{-1}{\gamma^{2}}\right)
$$

where $\mathbf{F}(\cdot \mid \cdot)$ is the incomplete elliptic integral of the first kind. From the latter invariant (C.2) we can read off the critical Keldysh parameter $\tilde{\gamma}^{\text {crit }}=\gamma / \operatorname{arcsinh}(\gamma)$ computed in (8.12). Using the modified invariant $a$ (C.2), the LMA condition (2.6) becomes

$$
\frac{m}{\omega}\left(-i 4 \mathbf{F}\left(i \frac{\gamma}{\tilde{\gamma}} \mid \frac{-1}{\gamma^{2}}\right)\right) \gg 1
$$

This quantity we have plotted in Fig. 20 versus $\gamma$ and different fixed combined Keldysh parameter $\tilde{\gamma}$. Very large $\tilde{\gamma}$ are excluded because of the LMA condition. Using the equation (5.15), we obtain the stationary worldline action

$$
\mathcal{W}_{0} \approx \frac{m^{2}}{e E} \frac{4}{\gamma}\left(-i \mathbf{E}\left(i \frac{\gamma}{\tilde{\gamma}} \mid \frac{-1}{\gamma^{2}}\right)\right)
$$




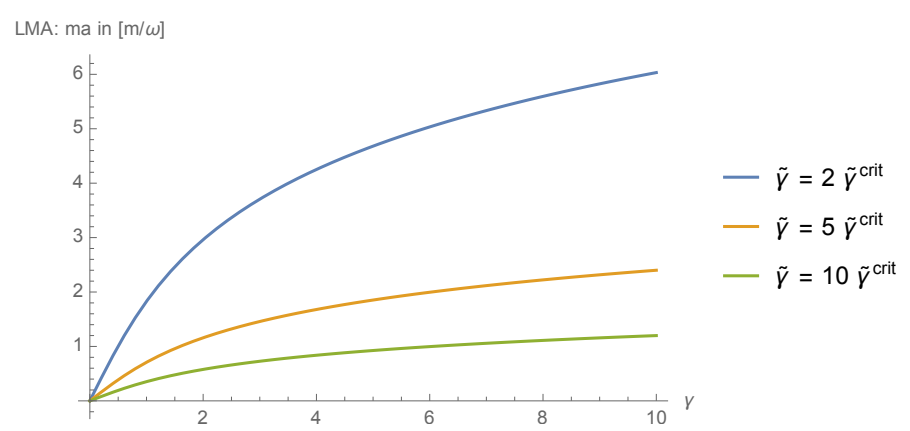

Figure 20. Relevant quantity ma expressed in units of $\frac{m}{\omega}$. The LMA requires $m a \gg 1$. Curves are plotted for different $\tilde{\gamma}$. The values are depicted in the plot legend. The critical combined Keldysh parameter is given in (8.12).

The function $\mathbf{E}(\cdot \cdot)$ denotes the incomplete elliptic integral of the second kind. The instanton solutions for the sinusoidal field are known [61]. Based on those, we can write the present modified solution in the right half plane, i.e. $u \in[-1 / 4,1 / 4]$, as

$$
\begin{aligned}
& x_{4}=\frac{m}{e E} \frac{1}{\gamma} \operatorname{arcsinh}\left(\frac{\gamma}{\sqrt{1+\gamma^{2}}} \mathbf{s d}\left(-i \mathbf{F}\left(i \frac{\gamma}{\tilde{\gamma}} \mid \frac{-1}{\gamma^{2}}\right) \frac{\sqrt{1+\gamma^{2}}}{\gamma} u \mid \frac{\gamma^{2}}{1+\gamma^{2}}\right)\right), \\
& x_{3}=\frac{m}{e E} \frac{1}{\gamma} \arcsin \left(\frac{\gamma}{\sqrt{1+\gamma^{2}}} \mathbf{c d}\left(-i \mathbf{F}\left(i \frac{\gamma}{\tilde{\gamma}} \mid \frac{-1}{\gamma^{2}}\right) \frac{\sqrt{1+\gamma^{2}}}{\gamma} u \mid \frac{\gamma^{2}}{1+\gamma^{2}}\right)\right)-\mathcal{C} .
\end{aligned}
$$

The functions $\mathbf{s d}(\cdot \mid \cdot)$ and $\mathbf{c d}(\cdot \mid \cdot)$ denote Jacobi elliptic functions. The shifting constant along the $\hat{x}_{3}$ axis is again determined by

$$
\mathcal{C}=x_{3}(u= \pm 1 / 4)
$$

The action above applies only for $\tilde{\gamma} \geq \tilde{\gamma}^{\text {crit }}$. Taking into account the case when the contribution of the weak field is absent, we can write the complete stationary worldline action as

$$
\mathcal{W}_{0}= \begin{cases}\frac{m^{2}}{e E} \frac{4}{\gamma}\left(-i \mathbf{E}\left(i \frac{\gamma}{\tilde{\gamma}} \mid \frac{-1}{\gamma^{2}}\right)\right) & \tilde{\gamma} \geq \tilde{\gamma}^{\text {crit }} \\ 4 \frac{m^{2}}{e E} \frac{\sqrt{\gamma^{2}+1}}{\gamma^{2}}\left(\mathbf{K}\left(\frac{\gamma^{2}}{\gamma^{2}+1}\right)-\mathbf{E}\left(\frac{\gamma^{2}}{\gamma^{2}+1}\right)\right) & \tilde{\gamma}<\tilde{\gamma}^{\text {crit }}\end{cases}
$$

Here, $\mathbf{K}(\cdot)$ and $\mathbf{E}(\cdot)$ denote the complete elliptic integrals of the first and second kind, respectively.

The analytical result (C.8) for the action is plotted in Fig. 21. Setting $\tilde{\gamma}=\tilde{\gamma}^{\text {crit }} 5 / 3$, we compare between the analytical prediction and the exact numerical computation. Both results do perfectly coincide as long $\epsilon \ll 1$. Note that this is the valid regime in the reflection picture. Only if we apply relatively large values $\epsilon=\{0.1,1.0\}$, there appears a notable difference between both curves. The effect of the weak field is well indicated. A considerable decrease applies in contrast to the situation with $\tilde{\gamma}=\tilde{\gamma}^{\text {crit }}$, where the weak field contribution is absent. As one would expect, for $\gamma=0(\Rightarrow \tilde{\gamma}=5 / 3)$ we find again the result from Fig. 5 . 

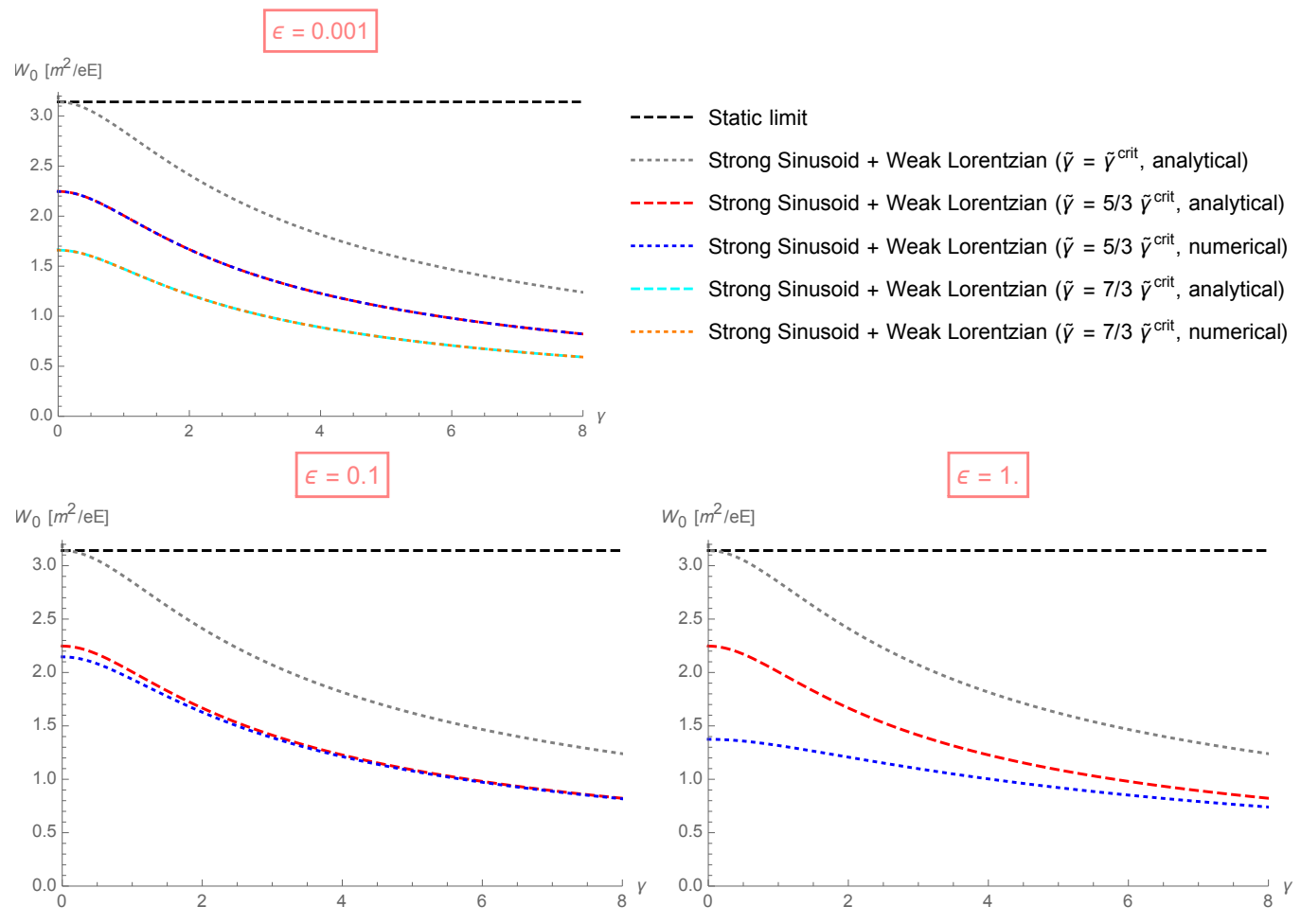

Figure 21. Stationary worldline action (C.8) for the strong sinusoidal field superimposed with a weak Lorentzian field (7.3) plotted versus $\gamma$. The analytical prediction is compared with exact numerical computations. The ratio between the strong and weak field strengths is set to $\epsilon=$ $\left\{10^{-3}, 10^{-1}, 10^{0}\right\}$ (top,bottom-left,bottom-right). The values for the combined Keldysh parameter are given in the plot legend with $\tilde{\gamma}^{\text {crit }}$ being computed via (8.12).
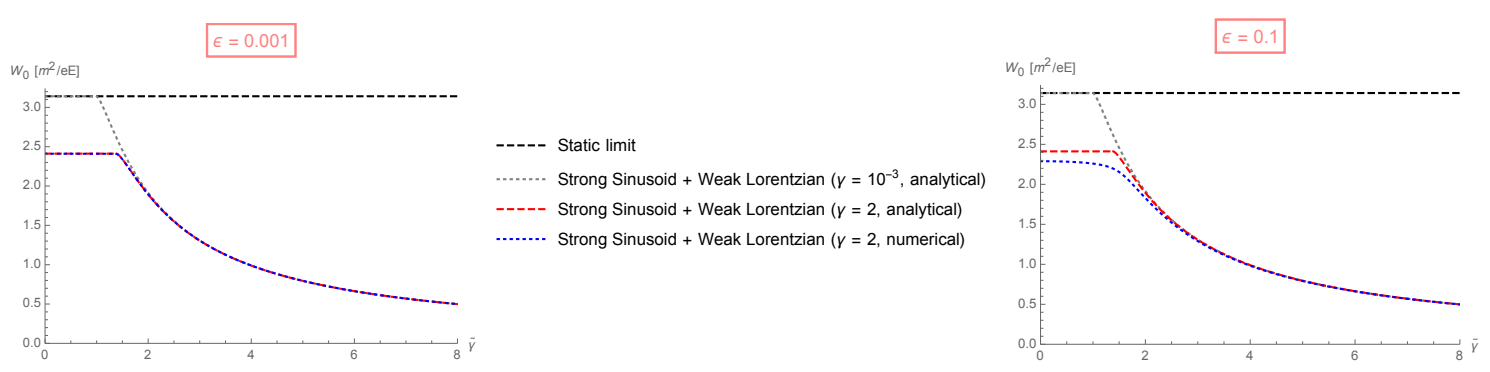

Figure 22. Same stationary worldline action as in Fig. 21 plotted versus the combined Keldysh parameter $\tilde{\gamma}$. The corresponding strong field inhomogeneities $\gamma$ as well as the ratios $\epsilon$ between the field strengths are given in the plots. The gray dotted curve is the known result in Fig. 5 (i.e. green curve).

We have seen that the superposition of a strong field with non-negligible Keldysh parameter and a weak fast field leads to an enhancement. However, this is only operative for $\tilde{\gamma}$ below the threshold $\tilde{\gamma}^{\text {crit }}$. For larger values it is again the weak rapid field that mainly drives the enhanced vacuum decay, cf. Fig. 22.

We should note, that in the latter described situation we have set the strong field 
inhomogeneity parameter as $\gamma=2$. Technically, a very strong field (Extreme Light Infrastructure, ELI) with high frequency (European XFEL) is neither realisable with current experimental facilities nor visioned for upcoming setups. One can alternatively think of the following realistic situation. Assume we start with a very strong field with strength $E_{1}$ and frequency $\omega_{1} \rightarrow 0$. Superimposing this low-frequent field with a second weak more rapid field, i.e. $E_{2} / E_{1} \ll 1$ and $\omega_{2} \gg \omega_{1}$, resembles the strong field setup depicted in Fig. 19.

\section{Additional figures}

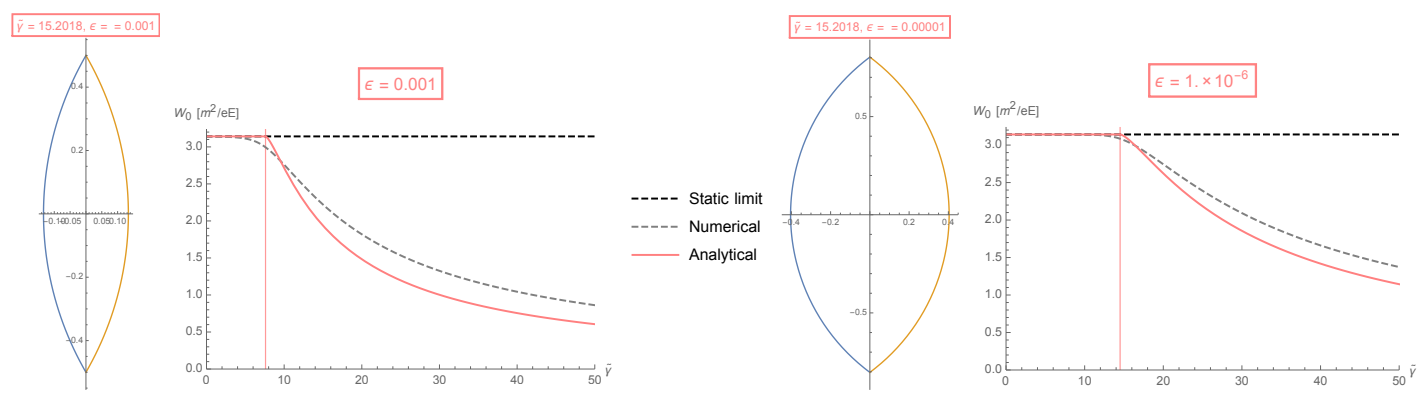

Figure 23. $\mathcal{W}_{0}$ for the weak sinusoidal field (7.3). The approximate analytical prediction (7.8) is compared with the exact numerical result for $\epsilon=\left\{10^{-3}, 10^{-6}\right\}$ (left,right). We have set $x_{4}^{\mathrm{p}}=x_{4}^{\mathrm{i}}$. Instanton paths are plotted for $\tilde{\gamma}=2 \tilde{\gamma}^{\text {crit }}(\epsilon=0.001)$ for $\epsilon=\left\{10^{-3}, 10^{-5}\right\}$ (left,right).
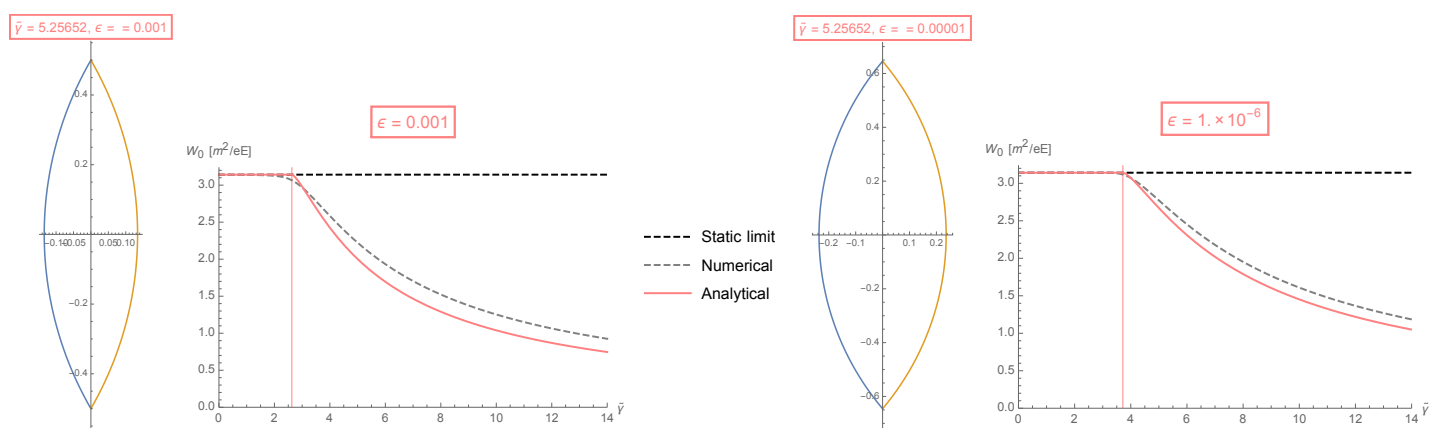

Figure 24. $\mathcal{W}_{0}$ for the weak Gaussian field (7.9). The approximate analytical prediction (7.14) is compared with the numerical result for $\epsilon=\left\{10^{-3}, 10^{-6}\right\}$ (left,right). We have set $x_{4}^{\mathrm{p}}=x_{4}^{\mathrm{i}}$. Instanton paths are plotted for $\tilde{\gamma}=2 \tilde{\gamma}^{\text {crit }}(\epsilon=0.001)$ for $\epsilon=\left\{10^{-3}, 10^{-5}\right\}$ (left,right).

\section{References}

[1] J. S. Schwinger, On gauge invariance and vacuum polarization, Phys. Rev. 82 (1951) 664-679.

[2] G. V. Dunne, H. Gies, and R. Schützhold, Catalysis of Schwinger Vacuum Pair Production, Phys. Rev. D80 (2009) 111301, [arXiv:0908.0948].

[3] G. V. Dunne, New Strong-Field QED Effects at ELI: Nonperturbative Vacuum Pair Production, Eur. Phys. J. D55 (2009) 327-340, [arXiv:0812.3163]. 
[4] F. Gelis and N. Tanji, Schwinger mechanism revisited, Prog. Part. Nucl. Phys. 87 (2016) 1-49, [arXiv: 1510.0545].

[5] H. Gies, Strong laser fields as a probe for fundamental physics, Eur. Phys. J. D55 (2009) 311-317, [arXiv:0812.0668].

[6] R. Schützhold, H. Gies, and G. Dunne, Dynamically assisted Schwinger mechanism, Phys. Rev. Lett. 101 (2008) 130404, [arXiv:0807.0754].

[7] A. Nuriman, B.-S. Xie, Z.-L. Li, and D. Sayipjamal, Enhanced electron-positron pair creation by dynamically assisted combinational fields, Phys. Lett. B717 (2012) 465-469.

[8] I. Akal, S. Villalba-Chávez, and C. Müller, Electron-positron pair production in a bifrequent oscillating electric field, Phys. Rev. D90 (2014), no. 11 113004, [arXiv:1409.1806].

[9] A. Otto, D. Seipt, D. Blaschke, B. Kämpfer, and S. A. Smolyansky, Lifting shell structures in the dynamically assisted Schwinger effect in periodic fields, Phys. Lett. B740 (2015) 335-340, [arXiv: 1412.0890].

[10] A. Otto, D. Seipt, D. Blaschke, S. A. Smolyansky, and B. Kämpfer, Dynamical Schwinger process in a bifrequent electric field of finite duration: survey on amplification, Phys. Rev. D91 (2015), no. 10 105018, [arXiv:1503.0867].

[11] M. F. Linder, C. Schneider, J. Sicking, N. Szpak, and R. Schützhold, Pulse shape dependence in the dynamically assisted Sauter-Schwinger effect, Phys. Rev. D92 (2015), no. 8 085009, [arXiv: 1505.0568].

[12] S. S. Bulanov, V. D. Mur, N. B. Narozhny, J. Nees, and V. S. Popov, Multiple colliding electromagnetic pulses: a way to lower the threshold of $e^{+} e^{-}$pair production from vacuum, Phys. Rev. Lett. 104 (2010) 220404, [arXiv:1003.2623].

[13] C. Kohlfürst, M. Mitter, G. von Winckel, F. Hebenstreit, and R. Alkofer, Optimizing the pulse shape for Schwinger pair production, Phys. Rev. D88 (2013) 045028, [arXiv: 1212.1385].

[14] F. Hebenstreit and F. Fillion-Gourdeau, Optimization of Schwinger pair production in colliding laser pulses, Phys. Lett. B739 (2014) 189-195, [arXiv:1409.7943].

[15] F. Hebenstreit, The inverse problem for Schwinger pair production, Phys. Lett. B753 (2016) 336-340, [arXiv: 1509.0869].

[16] F. Fillion-Gourdeau, F. Hebenstreit, D. Gagnon, and S. MacLean, Pulse shape optimization for electron-positron production in rotating fields, arXiv:1704.0891.

[17] N. Tanji, Dynamical view of pair creation in uniform electric and magnetic fields, Annals Phys. 324 (2009) 1691-1736, [arXiv:0810.4429].

[18] C. K. Dumlu, Multidimensional quantum tunneling in the Schwinger effect, Phys. Rev. D93 (2016), no. 6 065045, [arXiv:1507.0700].

[19] P. Copinger and K. Fukushima, Spatially Assisted Schwinger Mechanism and Magnetic Catalysis, Phys. Rev. Lett. 117 (2016), no. 8 081603, [arXiv:1605.0595]. [Erratum: Phys. Rev. Lett.118,no.9,099903(2017)].

[20] P. Elmfors and B.-S. Skagerstam, Electromagnetic fields in a thermal background, Physics Letters B 348 (1995), no. 1141 - 148.

[21] H. Gies, QED effective action at finite temperature: Two loop dominance, Phys. Rev. D61 (2000) 085021, [hep-ph/9909500]. 
[22] S. P. Kim, H. K. Lee, and Y. Yoon, Schwinger Pair Production at Finite Temperature in QED, Phys. Rev. D79 (2009) 045024, [arXiv: 0811.0349].

[23] W. Dittrich and H. Gies, Probing the quantum vacuum. Perturbative effective action approach in quantum electrodynamics and its application, Springer Tracts Mod. Phys. 166 (2000) 1-241.

[24] O. Gould and A. Rajantie, Thermal Schwinger pair production at arbitrary coupling, arXiv: 1704.0480.

[25] I. Akal, H. Gies and F. Karbstein (in preparation).

[26] M. I. Katsnelson and G. E. Volovik, Quantum electrodynamics with anisotropic scaling: Heisenberg-Euler action and Schwinger pair production in the bilayer graphene, JETP Lett. 95 (2012) 411-415, [arXiv:1203.1578]. [Pisma Zh. Eksp. Teor. Fiz.95,457(2012)].

[27] M. A. Zubkov, Schwinger pair creation in multilayer graphene, Pisma Zh. Eksp. Teor. Fiz. 95 (2012) 540, [arXiv:1204.0138].

[28] I. Akal, R. Egger, C. Müller, and S. Villalba-Chávez, Low-dimensional approach to pair production in an oscillating electric field: Application to bandgap graphene layers, Phys. Rev. D93 (2016), no. 11 116006, [arXiv:1602.0831].

[29] I. Akal, R. Egger, C. Müller and S. Villalba-Chávez (in preparation).

[30] F. Fillion-Gourdeau, D. Gagnon, C. Lefebvre, and S. MacLean, Time-domain quantum interference in graphene, Phys. Rev. B94 (2016), no. 12125423.

[31] F. Fillion-Gourdeau and S. MacLean, Time-dependent pair creation and the Schwinger mechanism in graphene, Phys. Rev. B92 (2015), no. 3035401.

[32] T. Oka and H. Aoki, Ground-state decay rate for the zener breakdown in band and mott insulators, Phys. Rev. Lett. 95 (Sep, 2005) 137601.

[33] M. F. Linder and R. Schützhold, Analog Sauter-Schwinger effect in semiconductors, arXiv: 1503.0710

[34] R. A. Abramchuk and M. A. Zubkov, Schwinger pair creation in Dirac semimetals in the presence of external magnetic and electric fields, Phys. Rev. D94 (2016), no. 11 116012, [arXiv: 1605.0237].

[35] A. Casher, H. Neuberger, and S. Nussinov, Chromoelectric Flux Tube Model of Particle Production, Phys. Rev. D20 (1979) 179-188.

[36] G. W. Semenoff and K. Zarembo, Holographic Schwinger Effect, Phys. Rev. Lett. 107 (2011) 171601, [arXiv:1109.2920].

[37] S. Bolognesi, F. Kiefer, and E. Rabinovici, Comments on Critical Electric and Magnetic Fields from Holography, JHEP 01 (2013) 174, [arXiv:1210.4170].

[38] Y. Sato and K. Yoshida, Potential Analysis in Holographic Schwinger Effect, JHEP 08 (2013) 002, [arXiv:1304.7917].

[39] K. Hashimoto, T. Oka, and A. Sonoda, Magnetic instability in AdS/CFT: Schwinger effect and Euler-Heisenberg Lagrangian of supersymmetric QCD, JHEP 06 (2014) 085, [arXiv: 1403.6336].

[40] K. Bitaghsir Fadafan and F. Saiedi, Holographic Schwinger effect in non-relativistic backgrounds, Eur. Phys. J. C75 (2015), no. 12 612, [arXiv:1504.0243]. 
[41] D. Kawai, Y. Sato, and K. Yoshida, Schwinger pair production rate in confining theories via holography, Phys. Rev. D89 (2014), no. 10 101901, [arXiv:1312.4341].

[42] Y. Sato and K. Yoshida, Holographic description of the Schwinger effect in electric and magnetic fields, JHEP 04 (2013) 111, [arXiv: 1303.0112].

[43] Y. Sato and K. Yoshida, Universal aspects of holographic Schwinger effect in general backgrounds, JHEP 12 (2013) 051, [arXiv:1309.4629].

[44] Y. Sato and K. Yoshida, Holographic Schwinger effect in confining phase, JHEP 09 (2013) 134, [arXiv: 1306.5512].

[45] D. D. Dietrich, Worldline holographic Schwinger effect, Phys. Rev. D90 (2014), no. 4045024 , [arXiv: 1405.0487].

[46] K. Hashimoto, T. Oka, and A. Sonoda, Electromagnetic instability in holographic QCD, JHEP 06 (2015) 001, [arXiv: 1412.4254].

[47] M. Ghodrati, Schwinger Effect and Entanglement Entropy in Confining Geometries, Phys. Rev. D92 (2015), no. 6 065015, [arXiv:1506.0855].

[48] I. Akal and D. D. Dietrich (to appear).

[49] J. Sonner, Holographic Schwinger Effect and the Geometry of Entanglement, Phys. Rev. Lett. 111 (2013), no. 21 211603, [arXiv: 1307.6850].

[50] W. Fischler, P. H. Nguyen, J. F. Pedraza, and W. Tangarife, Holographic Schwinger effect in de Sitter space, Phys. Rev. D91 (2015), no. 8 086015, [arXiv:1411.1787].

[51] I. A. Aleksandrov, G. Plunien, and V. M. Shabaev, Pulse shape effects on the electron-positron pair production in strong laser fields, Phys. Rev. D95 (2017), no. 5 056013, [arXiv:1701.0105].

[52] Z. Bern and D. A. Kosower, The Computation of loop amplitudes in gauge theories, Nucl. Phys. B379 (1992) 451-561.

[53] M. J. Strassler, Field theory without Feynman diagrams: One loop effective actions, Nucl. Phys. B385 (1992) 145-184, [hep-ph/9205205].

[54] C. Schubert, Perturbative quantum field theory in the string inspired formalism, Phys. Rept. 355 (2001) 73-234, [hep-th/0101036].

[55] I. K. Affleck, O. Alvarez, and N. S. Manton, Pair Production at Strong Coupling in Weak External Fields, Nucl. Phys. B197 (1982) 509-519.

[56] W. Dittrich and M. Reuter, Effective Lagrangians In Quantum Electrodynamics, Lect. Notes Phys. 220 (1985) 1-244.

[57] Y. Nambu, The use of the Proper Time in Quantum Electrodynamics, Prog. Theor. Phys. 5 (1950) 82-94.

[58] R. P. Feynman, Mathematical formulation of the quantum theory of electromagnetic interaction, Phys. Rev. 80 (1950) 440-457.

[59] R. P. Feynman, An Operator calculus having applications in quantum electrodynamics, Phys. Rev. 84 (1951) 108-128.

[60] G. V. Dunne, Heisenberg-Euler effective Lagrangians: Basics and extensions, in From fields to strings: Circumnavigating theoretical physics. Ian Kogan memorial collection (3 volume set) (M. Shifman, A. Vainshtein, and J. Wheater, eds.), pp. 445-522. 2004. hep-th/0406216. 
[61] G. V. Dunne and C. Schubert, Worldline instantons and pair production in inhomogeneous fields, Phys. Rev. D72 (2005) 105004, [hep-th/0507174].

[62] G. V. Dunne, Q.-h. Wang, H. Gies, and C. Schubert, Worldline instantons. II. The Fluctuation prefactor, Phys. Rev. D73 (2006) 065028, [hep-th/0602176].

[63] D. D. Dietrich and G. V. Dunne, Gutzwiller's trace formula and vacuum pair production, J. Phys. A40 (2007) F825-F830, [arXiv:0706.4006].

[64] C. Schneider and R. Schützhold, Prefactor in the dynamically assisted Sauter-Schwinger effect, Phys. Rev. D94 (2016), no. 8 085015, [arXiv: 1603.0086].

[65] I. Akal et al. (to appear).

[66] E. Strobel and S.-S. Xue, Semiclassical pair production rate for time-dependent electrical fields with more than one component: WKB-approach and world-line instantons, Nucl. Phys. B886 (2014) 1153-1176, [arXiv:1312.3261].

[67] J. Gordon and G. W. Semenoff, World-line instantons and the Schwinger effect as a Wentzel-Kramers-Brillouin exact path integral, J. Math. Phys. 56 (2015) 022111, [arXiv: 1407.0987].

[68] V. S. Popov, Pair production in a variable external field (quasiclassical approximation), Sov. Phys. JETP. 34 (1972) 709-718. [Zh. Eksp. Teor. Fiz.61,1334(1971)].

[69] E. Brezin and C. Itzykson, Pair production in vacuum by an alternating field, Phys. Rev. D 2 (Oct, 1970) 1191-1199.

[70] M. S. Marinov and V. S. Popov, Electron-Positron Pair Creation from Vacuum Induced by Variable Electric Field, Fortsch. Phys. 25 (1977) 373-400.

[71] D. D. Dietrich, Fermion production in time dependent fields, Phys. Rev. D68 (2003) 105005, [hep-th/0302229].

[72] H. Gies and K. Klingmüller, Pair production in inhomogeneous fields, Phys. Rev. D72 (2005) 065001, [hep-ph/0505099].

[73] F. Hebenstreit, R. Alkofer, and H. Gies, Pair Production Beyond the Schwinger Formula in Time-Dependent Electric Fields, Phys. Rev. D78 (2008) 061701, [arXiv:0807.2785].

[74] M. V. Fedorov, L. v. keldysh's "ionization in the field of a strong electromagnetic wave" and modern physics of atomic interaction with a strong laser field, Journal of Experimental and Theoretical Physics 122 (2016), no. 3 449-455.

[75] F. Hebenstreit, R. Alkofer, and H. Gies, Schwinger pair production in space and time-dependent electric fields: Relating the Wigner formalism to quantum kinetic theory, Phys. Rev. D82 (2010) 105026, [arXiv:1007.1099].

[76] F. Hebenstreit, R. Alkofer, and H. Gies, Particle self-bunching in the Schwinger effect in spacetime-dependent electric fields, Phys. Rev. Lett. 107 (2011) 180403, [arXiv:1106.6175].

[77] C. Schneider and R. Schützhold, Dynamically assisted Sauter-Schwinger effect in inhomogeneous electric fields, JHEP 02 (2016) 164, [arXiv:1407.3584].

[78] A. Ilderton, Localisation in worldline pair production and lightfront zero-modes, JHEP 09 (2014) 166, [arXiv:1406.1513].

[79] A. Ilderton, G. Torgrimsson, and J. Wardh, Nonperturbative pair production in interpolating fields, Phys. Rev. D92 (2015), no. 6 065001, [arXiv:1506.0918]. 
[80] I. A. Aleksandrov, G. Plunien, and V. M. Shabaev, Electron-positron pair production in external electric fields varying both in space and time, Phys. Rev. D94 (2016), no. 6065024 , [arXiv: 1606.0631].

[81] S. Lebedev and V. Ritus, Virial representation of the imaginary part of the lagrangian of an electromagnetic field, Zh. Eksp. Teor. Fiz. 86 (1984), no. 2 408-422.

[82] T. D. Cohen and D. A. McGady, The Schwinger mechanism revisited, Phys. Rev. D78 (2008) 036008, [arXiv:0807.1117].

[83] F. Hebenstreit, R. Alkofer, G. V. Dunne, and H. Gies, Momentum signatures for Schwinger pair production in short laser pulses with sub-cycle structure, Phys. Rev. Lett. 102 (2009) 150404, [arXiv:0901.2631].

[84] C. K. Dumlu and G. V. Dunne, Interference Effects in Schwinger Vacuum Pair Production for Time-Dependent Laser Pulses, Phys. Rev. D83 (2011) 065028, [arXiv:1102.2899].

[85] M. Orthaber, F. Hebenstreit, and R. Alkofer, Momentum Spectra for Dynamically Assisted Schwinger Pair Production, Phys. Lett. B698 (2011) 80-85, [arXiv:1102.2182].

[86] N. Abdukerim, Z.-L. Li, and B.-S. Xie, Effects of laser pulse shape and carrier envelope phase on pair production, Phys. Lett. B726 (2013) 820-826.

[87] C. K. Dumlu and G. V. Dunne, Complex Worldline Instantons and Quantum Interference in Vacuum Pair Production, Phys. Rev. D84 (2011) 125023, [arXiv:1110.1657].

[88] A. Ringwald, Pair production from vacuum at the focus of an X-ray free electron laser, Phys. Lett. B510 (2001) 107-116, [hep-ph/0103185].

[89] E. Akkermans and G. V. Dunne, Ramsey Fringes and Time-domain Multiple-Slit Interference from Vacuum, Phys. Rev. Lett. 108 (2012) 030401, [arXiv:1109.3489].

[90] Z. L. Li, D. Lu, B. S. Xie, L. B. Fu, J. Liu, and B. F. Shen, Enhanced pair production in strong fields by multiple-slit interference effect with dynamically assisted Schwinger mechanism, Phys. Rev. D89 (2014), no. 9093011.

[91] I. Akal et al. (in preparation). 